\title{
Dimensionally Restricted Causal Set Quantum Gravity: Examples in Two and Three Dimensions
}

\author{
William J. Cunningham ${ }^{1}$ and Sumati Surya ${ }^{2}$ \\ ${ }^{1}$ Perimeter Institute for Theoretical Physics, 31 Caroline St. N., Waterloo, Ontario, Canada \\ ${ }^{2}$ Raman Research Institute, CV Raman Ave., Sadashivanagar, Bangalore, 560080, India
}

\begin{abstract}
We study dimensionally restricted non-perturbative causal set quantum dynamics in 2 and 3 spacetime dimensions with non-trivial global spatial topology. The causal set sample space is generated from causal embeddings into spacetime lattices with global spatial topology $S^{1}$ and $T^{2}$ in 2 and 3 dimensions, respectively. The quantum gravity partition function over these sample spaces is studied using Markov Chain Monte Carlo (MCMC) simulations after analytic continuation. In both 2 and 3 dimensions we find a phase transition that separates the dominance of the action from that of the entropy. The action dominated phase is characterised by "layered" posets with a high degree of connectivity, while the causal sets in the entropy dominated phase are manifold-like. This phase transition is similar in character to that seen for the sample space of 2orders, which are topologically trivial, hence suggesting that this is a generic feature of dimensionally restricted sample spaces. The simulations use a newly developed framework for causal set MCMC calculations. Ours is the first implementation of a causal set dynamics restricted to 3 dimensions.
\end{abstract}




\section{Introduction}

Causal set theory (CST) makes the bold assumption that the spacetime continuum is an approximation of an underlying locally finite partially ordered set [1 7]. While the continuum approximation of the theory is deeply rooted in Lorentzian geometry [8, 9], there are fewer known constraints on the dynamics. In the most conservative approach, the partition function over Lorentzian geometries is replaced by the weighted sum over the sample space $\mathrm{E}^{1}$ $\Omega_{n}$ of $n$-element causal sets

$$
Z_{\Omega_{n}} \equiv \sum_{c \in \Omega_{n}} \mu(c)
$$

where $\mu(c) \in \mathbb{C}$ is the measure. Fixing $n$ is analogous to fixing the total spacetime volume in the spirit of unimodular gravity [10, 11], and is a natural choice for causal sets. In analogy with quantum field theory, one moreover expects that the measure takes the specific form

$$
\mu(c)=\exp \left(i S_{\mathrm{BD}}^{(\mathbf{d})}(c) / \hbar\right)
$$

where $S_{B D}^{(\mathbf{d})}(c)$ is the d-dimensional discrete Einstein-Hilbert or Benincasa-Dowker (BD) action [12 14].

Even in this most conservative setting, the dynamics encoded in $Z_{\Omega_{n}}$ is considerably more inclusive than in other discrete approaches to quantum gravity . For one, $\Omega_{n}$ is entropically dominated by causal sets that are nothing like any spacetime. These Kleitman-Rothschild (KR) posets have three layers or moments of "time" and their number grows at least like $\sim 2^{n^{2} / 4}[15$. There is moreover a hierarchy of sub-dominant posets which are also not manifold-like [16, 17]. The action must therefore be able to counter this entropy in the classical limit of the theory, where one expects the continuum to be emergent. $\Omega_{n}$ also contains causal sets that are approximated by spacetime geometries of any dimension, for large enough $n$. Thus, the action must additionally ensure that $\mathbf{d}=4$ dominates in the classical limit of the theory.

The choice of measure in the partition function $Z_{\Omega_{n}}$ is therefore very important. Although the measure Eqn. 2 appears natural from the perspective of local quantum field theory, there is no fundamental or order theoretic motivation for this particular choice. Since the continuum is replaced by the discretum of causal sets, with the space of causal sets including those that are not continuum-like, one expects the Einstein-Hilbert action to be an effective rather than a fundamental action (see for example [18]). Thus the measure could include more fundamental order theoretic contributions that cannot be guessed at from the continuum. An example of this is the weight coming from the number of possible relabellings of the causal set. In [19] such a counting measure was used to help suppress the contribution of the class of (non-continuum-like) bilayer posets to the partition function.

In particular, in the continuum the formal path integral of quantum gravity is defined over geometries of a fixed dimension $\mathbf{d}$ with the measure given in terms of the Einstein-Hilbert

\footnotetext{
${ }^{1}$ Refer to Appendix A for a list of standard definitions in CST.
} 
action of the same dimension. The choice of the continuum-inspired measure, Eqn. (2), therefore requires a choice of $\mathbf{d}$ from the outset. In order to mimic the formal continuum path integral, $\Omega_{n}$ should be further restricted to the set of $n$-element causal sets whose continuum limit, if it exists, is a $\mathbf{d}$ dimensional spacetime.

Even if it were possible to do this dimensional "cherry picking", the naive choice of measure, Eqn. (2), ignores order-theoretic factors that could arise from causal set theory. While the measure must satisfy discrete covariance or order invariance, the continuum can only act as a rough guide. For example, it is possible that due to the nature of the continuum approximation in CST (see [1 7]), there are relative weights between different continuumlike causal sets which cannot be obtained purely from a continuum theory. Also, since the sample space must contain causal sets that are not manifold-like, there can also be a relative weight between manifold-like and non-manifold-like causal sets. Importantly, hidden in the measure of Eqn. (1) is the choice of sample space, which may come with its own preferred choice of measure. Thus, in trying to mimic a continuum path integral in CST, a myriad choices can be made in principle.

Our aim in this work is to generate dimensional and topological restrictions $\Omega_{n}(M, g)$ of $\Omega_{n}$ associated with a continuum spacetime $(M, g)$ in order to study dimensionally restricted causal set dynamics. For a causal diamond $D_{2}$ in $\mathbf{d}=2$ Minkowski spacetime $\mathbb{M}^{2}$, it was shown that the continuum dimension coincides with a natural order-theoretic dimension defined for $d$-orders [20, 21]. These 2-orders (or 2d-orders) admit a simple representation in the light-cone coordinates, with the elements occupying sites in a regular light-cone lattice in $\left(D_{2}, \eta\right)$. While the sample space of $n$-element 2-orders does contain those that are not manifold-like, it is dominated by causal sets that are approximated by the flat causal diamond $\left(D_{2}, \eta\right)[22$, 23] with trivial global spatial topology. Entropically, however, it is a much easier beast to handle than $\Omega_{n}$ and hence it has been possible to do an extensive study of the continuum-inspired dynamics for 2-orders [21, 24, 25].

In higher dimensions, the $d$-orders do not correspond to causal sets that are continuumlike, since the associated "light-cones" for a $d$-order are in fact hypercubes, with cross-sections given by $(\mathbf{d}-1)$-simplices, rather than $(\mathbf{d}-1)$ spheres. Hence restricting to the sample space of $d$-orders would take us further away from our goal of mimicking the continuum path integral.

In order to make progress in finding a dimensional restriction for $\mathbf{d}>2$ and also to explore non-trivial global topology in $\mathbf{d} \geq 2$, it is helpful to view the 2 -orders as causal sets obtained from causal embeddings into the causal diamond $\left(D_{2}, \eta\right)$. Let $\Omega_{n}\left(D_{2}, \eta\right)$ denote the sample space of causal sets that causally embed into $\left(D_{2}, \eta\right)$. To generate $\Omega_{n}\left(D_{2}, \eta\right)$ on the computer, consider a regular light-cone lattice on $D_{2}$ with $m$ sites. Starting from an $n$-site filling of this lattice and using the induced causal relations between the sites in $\left(D_{2}, \eta\right)$, we obtain an $n$-element causal set which causally embeds into $\left(D_{2}, \eta\right)$. In the limit of large $m$, the set of causal sets obtained this way $\sim \Omega_{n}\left(D_{2}, \eta\right)$. In the specific case, with $m=n^{2}$, and such that no two filled sites have the same light-cone coordinates (i.e., they are not 
null related) we recover the sample space of 2-orders, which can therefore be viewed as an approximation of $\Omega_{n}\left(D_{2}, \eta\right)$.

In this work we propose such a lattice-inspired model for generating dimensionally and topologically restricted sample spaces $\Omega_{n}(M, g)$ from a topologically non-trivial but finite volume (region of a ) spacetime $(M, g)$. In Sec. 2 we describe how to obtain an approximation $\Omega^{(m, n)}$ of the sample space on the computer via a latticisation of $(M, g)$ into $m$-sites. We focus on two specific examples: (a) $\left(M_{a}, \eta_{a}\right)$ is the flat cylinder spacetime in $\mathbf{d}=2$, with global spatial topology $S^{1}$ and (b) $\left(M_{b}, \eta_{b}\right)$ is the flat toroidal spacetime in $\mathbf{d}=3$, with global spatial topology $T^{2}$. In order to generate $\Omega^{(m, n)}$ we use a lattice-gas inspired Markov Chain Monte Carlo (MCMC) algorithm to obtain the expectation values of various observables, or orderinvariants. We demonstrate that for large enough $m, \Omega_{a, b}^{(m, n)} \sim \Omega_{n}\left(M_{a, b}, \eta_{a, b}\right)$ by noting that the expectation values of various observables calculated in the MCMC simulations converge to the same values. In Sec. 3 we set up a continuum-inspired dynamics on $\Omega_{n}\left(M_{a, b}, \eta_{a, b}\right)$, with any extra order theoretic measure coming entirely from the choice of the sample space. The introduction of a parameter $\beta$ analogous to an inverse temperature admits an analytic continuation, so that the lattice-gas MCMC algorithms may be used to simulate the nonperturbative dynamics.

Section 4 contains the results of the lattice-gas MCMC simulations for $\left(M_{a, b}, \eta_{a, b}\right)$. We find a phase transition which exhibits the tussle between entropy and action in the Lorentzian statistical geometry setting ${ }^{2}$ which echoes that found for 2-orders [21]. In Section 5 we discuss some of the open questions and ongoing related work. This study sets the stage for a new class of investigations into causal set dynamics, which can bridge the gap between the deep quantum, order-theoretic regime and the framework of the continuum path integral. Finally, Appendix A contains some of the standard definitions used throughout this work, italicised when they first appear in the text for ease of reading, and Appendix B contains a calculation of the ordering fraction for causal set approximated by the cylinder spacetime.

\section{Generating new sample spaces $\Omega_{n}(M, g)$}

As mentioned in the introduction, the sample space $\Omega_{n}(M, g)$ of causal sets that causally embed into a finite volume (preferably globally hyperbolic) region $(M, g)$ of a $\mathbf{d}$-dimensional spacetime includes those that are non-manifold-like as well as those whose continuum approximation differs significantly from $(M, g)$. The only point of similarity for the manifold-like causal sets in $\Omega_{n}(M, g)$ is their global topology. A quantum dynamics on $\Omega_{n}(M, g)$ would thus be an example of a $\mathbf{d}$-dimensional causal set quantum gravity with global topology $M$. Our goal in this section is to see how to generate $\Omega_{n}(M, g)$ on the computer, using an appropriate latticisation of $(M, g)$.

To make the discussion concrete, we consider the flat spacetimes $\left(M_{\mathbf{d}}, \eta\right)$ with toroidal

\footnotetext{
${ }^{2}$ In [19] this has been studied for the quantum path integral for the specific case of bilayer posets.
} 

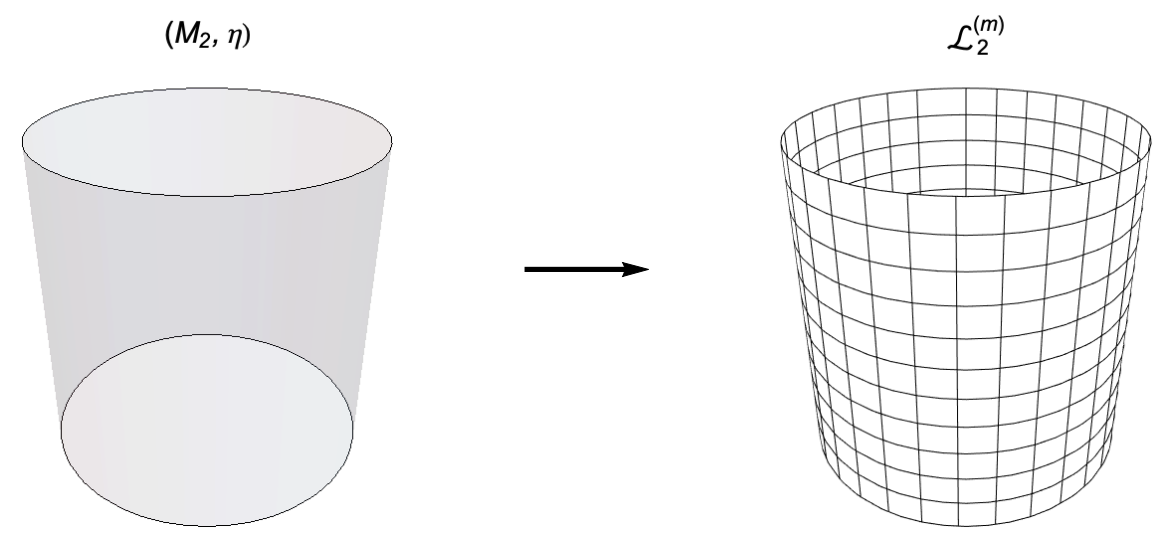

Figure 1: The latticisation $\mathcal{L}^{(m)}$ of the spacetime $\left(M_{a}, \eta\right)$ into a grid of height $h$ and width $w$, where $m=h \times w$.
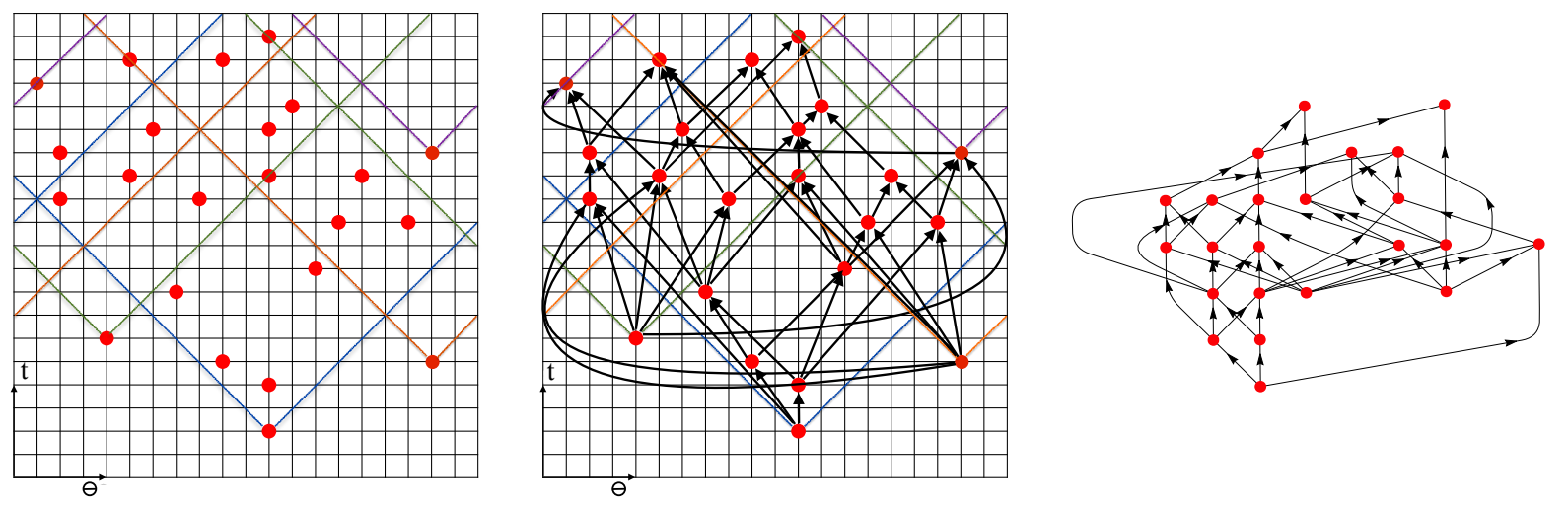

Figure 2: (i) An $n$-site filling of an $m$-site cylindrical lattice (the spatial edges are identified). The light-cones for a few of the elements are shown and can be seen to wrap around the cylinder. (ii) The links or irreducible relations between elements are obtained from the causal structure of the lattice. The curved lines denote the relations obtained by going "around" the cylinder, due to the topological non-triviality of the causal structure. (iii) The resulting Hasse diagram for the causal set.

global spatial topology

$$
\begin{aligned}
M_{\mathbf{d}} & \sim I \times T^{\mathbf{d}-1}=\mathbb{R} \times \underbrace{S^{1} \times \ldots S^{1}}_{\mathbf{d}-1} \\
\eta_{\mu \nu} d x^{\mu} d x^{\nu} & =-d t^{2}+\sum_{i=1}^{\mathbf{d}-1} d \theta_{i}^{2}, \quad \theta_{i}(\bmod ) 2 \pi, t \in[0, h] .
\end{aligned}
$$



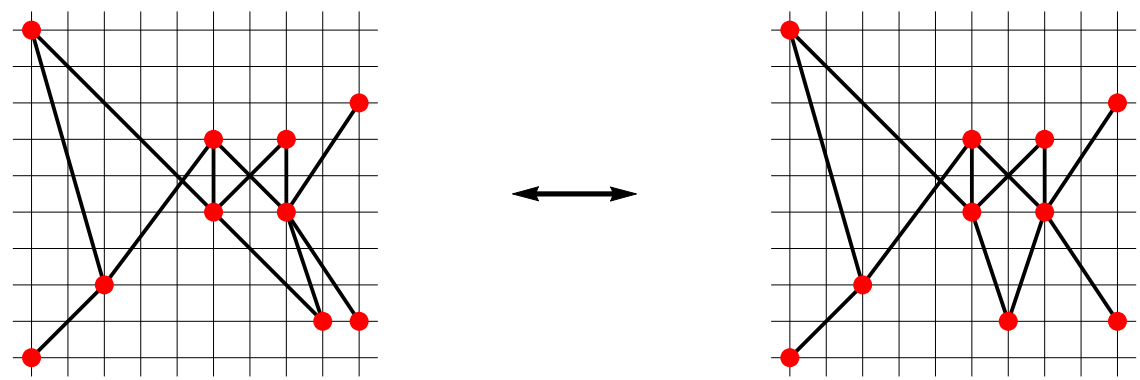

Figure 3: An example of two distinct $n$-site fillings of an $m$-lattice which produce the same causal set.

These spacetimes admit a natural latticisation $\mathcal{L}_{\mathbf{d}}^{(m)}$ into an $h \times w^{\mathbf{d}-1}=m$ site cylindrical lattice, with each lattice site having the coordinates $\left(t, \theta_{1}, \ldots, \theta_{\mathbf{d}-1}\right)$, where $t \in\{0,1, \ldots, h\}$ and $\theta_{i}=2 \pi k_{i} / w, k_{i} \in\{0,1, \ldots, w-1\}$, with the identification $\theta_{i} \sim \theta_{i}+2 \pi$ (see Fig. 1). The lattice is parametrized by $m$ and also the aspect ratio $\alpha=h / w$. Given the metric Eqn 3 , the local light cones are therefore at $45^{\circ}$.

For any $n$-site filling of $\mathcal{L}_{\mathbf{d}}^{(m)}$, one can construct a causal set using the causal relations in $\left(M_{\mathbf{d}}, \eta\right)$ induced on the $n$-sites as illustrated in Fig. 2. The causal future and past of every element is given via the metric Eqn 3. In Fig. 2 there are three minimal elements which have no elements to their past and three maximal elements with no elements to their future.

In order to accommodate both the $n$-element chain and the $n$-element antichain in the sample space, we let $n \leq w, h$ so that $m \geq n^{2}$. As discussed in the introduction, for the 2-orders, $m=n^{2}$ and the global topology is $\sim D_{2}$. Since we would like to capture the non-trivial global topology, we also require that $h>w$, to allow at least a few null rays to wrap around the cylinder once. Ideally, $h$ should be significantly larger than $w$ for the full global topology to manifest itself in the sample space.

Let $\mathcal{E}_{\mathbf{d}}^{(m, n)}$ be the set of all possible $n$-site fillings into $\mathcal{L}_{\mathbf{d}}^{(m)}$, so that $\left|\mathcal{E}_{\mathbf{d}}^{(m, n)}\right|=\left(\begin{array}{c}m \\ n\end{array}\right)$. Let $\Omega_{\mathbf{d}}^{(m, n)}$ denote the set of unlabelled causal sets, i.e., the $(m, n)$-orders obtained from $\mathcal{E}_{\mathbf{d}}^{(m, n)}$. For $n \ll m$, the map $\mathcal{E}_{\mathbf{d}}^{(m, n)} \rightarrow \Omega_{\mathbf{d}}^{(m, n)}$ is strictly non-injective since a small change in the location of a filled site in $\mathcal{L}_{\mathbf{d}}^{(m)}$ yields the same causal set in $\Omega_{\mathbf{d}}^{(m, n)}$ (Fig. 3). Each causal set in $\Omega_{\mathbf{d}}^{(m, n)}$ is therefore typically represented multiple times in $\mathcal{E}_{\mathbf{d}}^{(m, n)}$. For example, an $n$-element chain can be represented in multiple ways in $\mathcal{E}_{\mathbf{d}}^{(m, n)}$ as can an $n$-element antichain. However, the number of possible representations for each $c \in \Omega_{\mathbf{d}}^{(m, n)}$ is different. For a causal set obtained from a random selection of elements in $\mathcal{E}_{\mathbf{d}}^{(m, n)}$ the redundancy will be considerably smaller, since each element can occupy a smaller number of allowed sites without changing the causal relations. Thus, the map induces a non-uniform measure on $\Omega_{\mathbf{d}}^{(m, n)}$.

In general this measure depends not only on $n$, but also on $m$. As $m$ increases, however, the relative measures between the causal sets in $\Omega^{(m, n)}$ will become independent of $m$. Roughly, this is because the number of empty sites surrounding each filled site increases as 
the grid gets finer, but a move from a filled site to any one of these empty sites leaves the causal set unchanged. Thus the "degeneracy" of the representation of each $c \in \Omega^{(m, n)}$ in $\mathcal{E}^{(m, n)}$ should increase fairly uniformly with $m$. We give evidence for this below in Fig. 6 and also in Sec. 4 .

In particular, in the limit of $m \rightarrow \infty$, with the lattice grid size going to zero, we expect that $\Omega_{\mathbf{d}}^{(m, n)} \rightarrow \Omega_{n}\left(M_{\mathbf{d}}, \eta\right)$. Generating $\mathcal{E}_{\mathbf{d}}^{(m, n)}$ on the computer is therefore one way to obtain a reasonable approximation of $\Omega_{n}\left(M_{\mathbf{d}}, \eta\right)$, as long as $m$ is sufficiently large.

One can generate $\mathcal{E}_{\mathbf{d}}^{(m, n)}$ by "walking" through every possible $n$-site filling of $\mathcal{L}_{\mathbf{d}}^{(m)}$, but of course this is computationally expensive, since the number of possible fillings grows as $\sim m^{n}$. It is therefore useful to devise a Markov Chain move that will effectively do the same job. The move we describe is very simple and is inspired by lattice-gas models in statistical physics.

Consider an $n$-site filling $E \in \mathcal{E}_{\mathbf{d}}^{(m, n)}$ and let $c(E)$ be the associated causal set in $\Omega_{\mathbf{d}}^{(m, n)}$. Let $\bar{E}$ represent the set of $m-n$ unfilled sites. Pick one of the filled sites $e=(t, x) \in E$ and one of the unfilled sites $\bar{e}=(\bar{t}, \bar{x}) \in \bar{E}$ independently and at random, and swap them, to get the new filled site $e^{\prime}=(\bar{t}, \bar{x})$ and the new unfilled site $\bar{e}^{\prime}=(t, x)$. One has moved from $E$ to a different $n$-site filling $E^{\prime} \in \mathcal{E}_{\mathbf{d}}^{(m, n)}$ via the filled-empty exchange $e \leftrightarrow \bar{e}$. Fig. 4 shows an example of this lattice-gas move in $\mathbf{d}=2$. We can then use the standard MCMC methods to sample $\Omega_{\mathbf{d}}^{(m, n)}$ and calculate the expectation value of several order invariants.

In the above lattice-gas move, since $e$ and $\bar{e}$ are picked randomly in $E$ and $\bar{E}$, the possibility of emptying the filled site $e$ to an empty site in its vicinity is relatively small. Hence we expect that the resulting causal set $c^{\prime}\left(E^{\prime}\right)$ will in fact be distinct from $c(E)$. In Fig. 5 we have shown the results of an MCMC simulation ${ }^{3}$ for $\mathbf{d}=2, n=200, h=800, w=200$, where the order invariants (or observables) are the Benincasa-Dowker action $S_{\mathrm{BD}}^{(2)}$ as well as the ordering fraction plotted at every MCMC step. To ensure a large enough lattice height $h$ for capturing the effects of non-trivial global spatial topology, a choice of aspect ratio $\alpha=4$ is sufficient for our purposes. In Appendix $B$ we calculate the expectation value of the ordering fraction for a causal set that is approximated by the cylinder spacetime. For $\alpha=4$, this gives the value of $\sim 0.88$, which is what we observe in Figs. 5, 6. Even though two distinct causal sets can have the same values for these observables, the converse is not true, i.e., if the two sets of observables are different, then so are the causal sets. Hence it is clear that at almost every step one obtains a distinct causal set.

To establish the asymptotic regime when $\Omega^{(m, n)}$ becomes independent of $m$, we run simulations for different values of $m$ while keeping $n$ and $\alpha$ fixed. In Fig. 6 we demonstrate this for $\mathbf{d}=2, n=200, \alpha=4$ for different filling fractions $f=n / m$ or different values of $m=h \times w=\alpha \times w^{2}$. Here, instead of recording every step, we record every sweep, defined as $\left(\begin{array}{c}n \\ 2\end{array}\right)$ steps. From the plotted data, one sees a clear convergence of the thermalised configurations with $m$. Hence for $\mathbf{d}=2$, and these choices of $n$ and $\alpha, m=4 \times 10^{4}$ is

\footnotetext{
${ }^{3}$ We will describe the details of these simulations in Sec. 3 .
} 

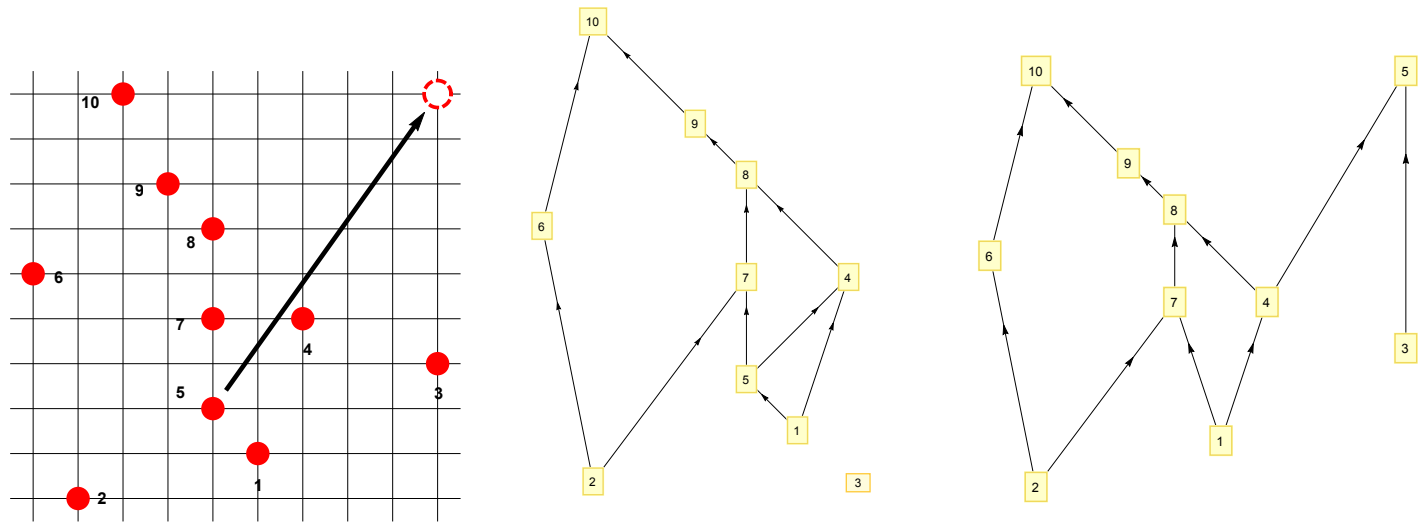

Figure 4: An example of the lattice-gas move. (i) A filled site is picked at random and moved to an unfilled site, which itself is chosen at random and independently. This move is typically non-local and can drastically change the associated causal set, shown here by (ii) $\rightarrow$ (iii).
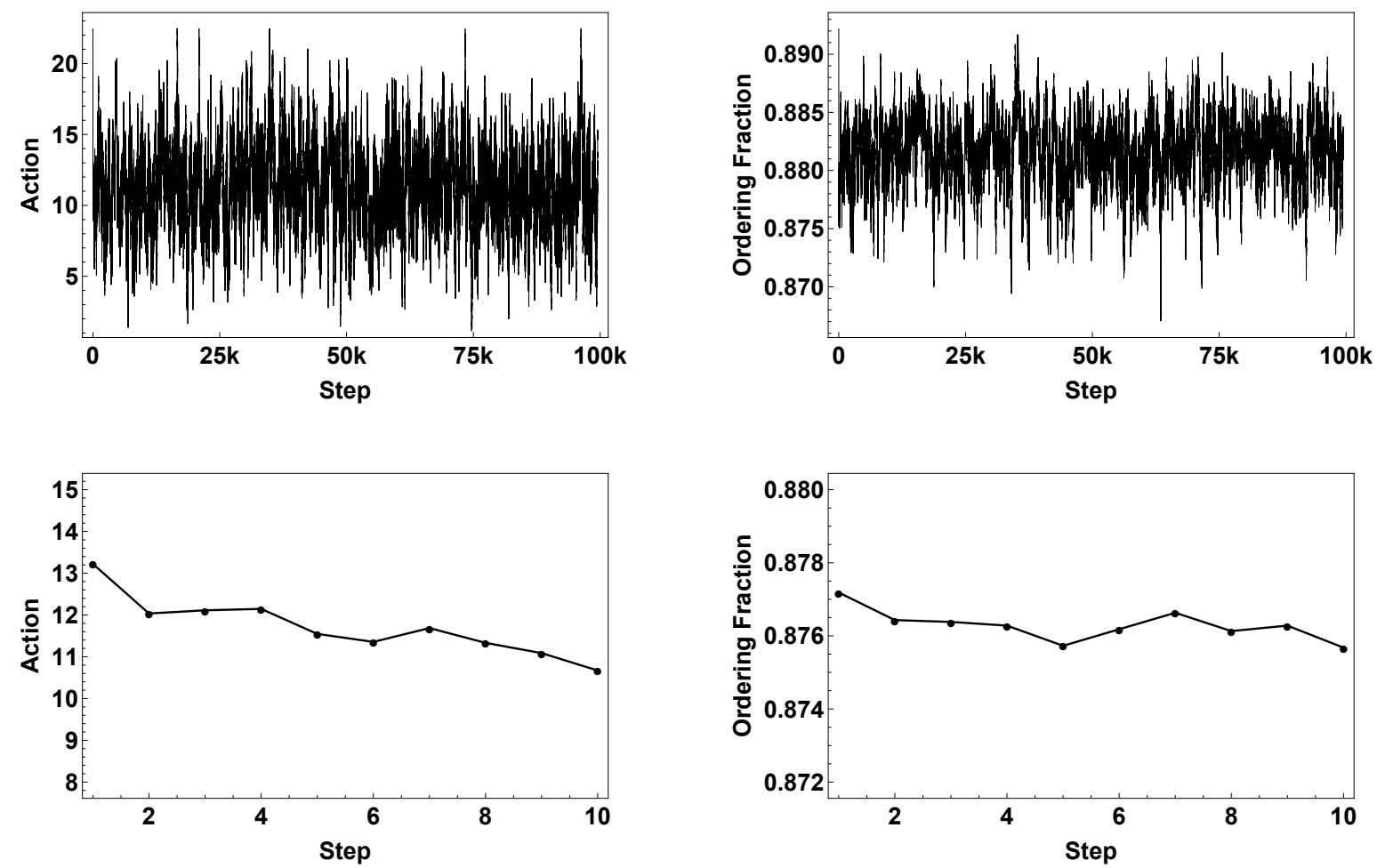

Figure 5: The action and ordering fraction are plotted at each step in the Markov chain, with the former calculated using the non-locality scale $\epsilon=0.1$. These observables vary at each step which implies that a different causal set is generated at each step. The top two panels show the variation over $10^{5}$ steps while the bottom two panels show just the first 10 steps. These values were measured for $\mathbf{d}=2$ using $n=w=200, h=800$, after thermalising for 1000 sweeps. 

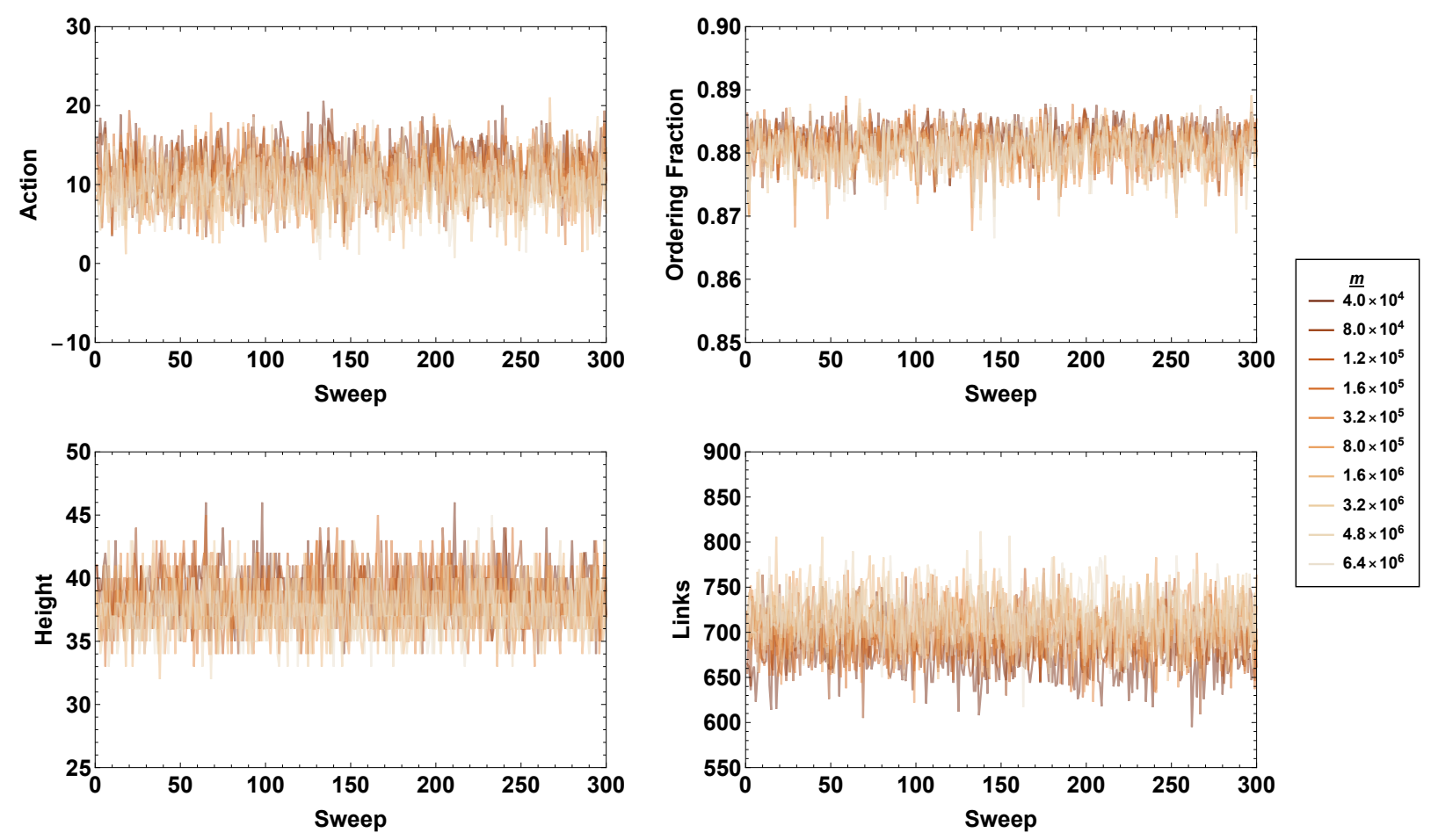

Figure 6: Raw data for the action, the ordering fraction, the height, and the number of links from lattice-gas MCMC simulations which generate the typical $(m, n)$-orders, for $\mathbf{d}=2$, $n=200$ and $\alpha=4$. When $m \gg n$, these order invariants can be seen to be independent of $m$.

sufficiently in the asymptotic regime for our purposes.

In the sample space $\Omega_{n}\left(M_{\mathbf{d}}, \eta\right)$, a special role is played by those causal sets that embed faithfully into, or are approximated by $\left(M_{\mathbf{d}}, \eta\right)$. An important question is how this class of causal sets is represented in $\Omega_{\mathbf{d}}^{(m, n)}$.

It was shown in [22, 23] that the typical 2-order is a random 2-order obtained by choosing the two linear orders (or light-cone $(u, v)$-coordinates) randomly and independently. It was then shown in [20] that the class of random orders are those that faithfully embed into $\left(D_{2}, \eta\right)$. In analogy, we define the random $(m, n)$-order to be that obtained from a random filling of $\mathcal{L}_{\mathbf{d}}^{(m)}$. Are these typical? We can answer this question at least partially by employing the MCMC algorithm on $\mathcal{E}_{\mathbf{d}}^{(m, n)}$, and comparing the expectation value of various observables with that of a random $(m, n)$-order. As we see from Figs. 7 where we have plotted the expectation values of the abundance of $r$-element intervals, one cannot distinguish a typical causal set in $\Omega_{\mathbf{d}}^{(m, n)}$ from a random $(m, n)$-order either in $\mathbf{d}=2$ or $\mathbf{d}=3$. We find the same when further comparing with the causal sets obtained from Poisson sprinklings into $\left(M_{\mathbf{d}}, \eta\right)$, Eqn. 3 . 

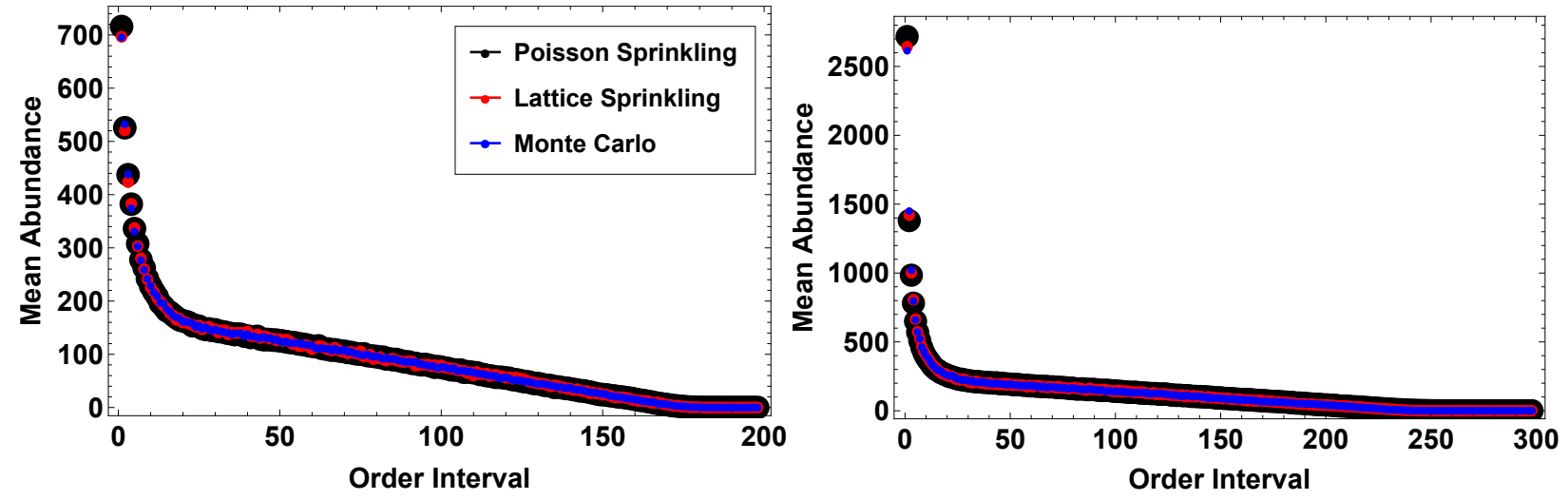

Figure 7: Mean interval abundance curves for $\mathbf{d}=2$ (left) and $\mathbf{d}=3$ (right). The three curves represent a Poisson sprinkling into the continuum, a random $(m, n)$-order sprinkled onto the lattice, and a typical $(m, n)$-order generated via a lattice-gas MCMC simulation.

\section{Setting up the Dynamics}

Given $\Omega_{n}\left(M_{\mathbf{d}}, \eta\right)$, we can write the dimensionally restricted path-sum for causal set quantum gravity as

$$
Z_{n}^{(\mathbf{d})}=\sum_{c \in \Omega_{\mathbf{d}}\left(M_{\mathbf{d}}, \eta\right)} \exp \left(i S_{\mathrm{BD}}^{(\mathbf{d})}(c) / \hbar\right)
$$

In order to evaluate the expectation values of covariant observables or order-invariants on the computer, we first introduce an analytic continuation parameter $\beta$ such that [21]

$$
Z_{n}^{(\mathbf{d})}(\beta)=\sum_{c \in \Omega_{n}(M, g)} \exp \left(i \beta S_{\mathrm{BD}}^{(\mathbf{d})}(c) / \hbar\right) \underset{\beta \rightarrow i \beta}{\longrightarrow} \mathcal{Z}_{n}^{(\mathbf{d})}(\beta)=\sum_{c \in \Omega_{n}(M, g)} \exp \left(-\beta S_{\mathrm{BD}}^{(\mathbf{d})}(c) / \hbar\right)
$$

This converts the quantum path-sum $Z_{n}^{(\mathbf{d})}(\beta)$ to a statistical partition function $\mathcal{Z}_{n}^{(\mathbf{d})}(\beta)$ over discrete Lorentzian geometries, which we can try to simulate on the computer.

The latticisation of $\left(M_{\mathbf{d}}, \eta\right)$ into $\mathcal{L}_{\mathbf{d}}^{(m)}$ with the associated sample space $\Omega_{\mathbf{d}}^{(m, n)}$ is the first step in this implementation, using which we define the discrete partition function

$$
\mathcal{Z}_{n, m}^{(\mathbf{d})}(\beta) \equiv \sum_{c \in \Omega_{\mathbf{d}}^{(m, n)}} \exp \left(-\beta S_{\mathrm{BD}}^{(\mathbf{d})}(c) / \hbar\right)
$$

We can then implement the MCMC lattice-gas move described above to perform a weighted walk through $\Omega_{\mathbf{d}}^{(m, n)}$, using the Metropolis algorithm, with the expectation values $\langle O\rangle$ of different order-invariants $O$ being calculated using importance sampling.

In simulations, the lattice $\mathcal{L}^{(m)}$ is represented by the integer sequence $L=\{0, \ldots, m-1\}$, where the entry $L_{i}$ represents an element $e$ with spacetime coordinates $t_{i}=\left\lfloor L_{i} / w\right\rfloor / h$ and 
$\theta_{i}=(2 \pi / w)\left(L_{i} \bmod w\right)^{4}$. To represent an $(m, n)$-order $E \in \mathcal{E}_{\mathbf{d}}^{(m, n)}$ on $\mathcal{L}_{\mathbf{d}}^{(m)}$, we select $n$ unique entries from $L$, or equivalently, the first $n$ entries of a random permutation of $L$. Thus, a random permutation of $L$ yields a random $(m, n)$-order. Moreover, a swap between a randomly chosen element in the first $n$ entries with a randomly and independently chosen element from the last $m-n$ entries, represents the lattice-gas move $e \leftrightarrow \bar{e}$. Because the lattice-gas move is typically non-local, every time we update $L$ we must also recalculate all causal relations using the spacetime coordinates $\left(t_{i}, \theta_{i}\right)$. The representation of the causal (adjacency) matrix for the corresponding causal set, as well as the implementation of the action calculation, are described in [26].

States are updated in the Markov chain according to the Metropolis rule: a new configuration resulting from a lattice-gas move is accepted if $\exp \left(-\beta \Delta S_{B D}^{(\mathbf{d})}\right)>\mathfrak{u}$, where $\mathfrak{u}$ is a uniform random variable in the range $[0,1)$. Observables are recorded each sweep, or $\left(\begin{array}{l}n \\ 2\end{array}\right)$ steps, and simulations are run for at least $10^{4}$ sweeps. Despite the compact data structures limiting memory usage $(\sim 10 \mathrm{MB})$ and parallel algorithms used to do calculations, it is prohibitively expensive to increase $n$ above $O\left(10^{2}\right)$ because each of the $10^{4}\left(\begin{array}{l}n \\ 2\end{array}\right)$ updates still requires $\sim 100 \mu \mathrm{sec}$ and the simulation has an overall complexity of $O\left(n^{5}\right)$.

For this reason, we have chosen to study $n=200,300$ in order to limit individual experiments to finish in under three days when running on machines with up to 56 cores. Due to the exceptionally high volume of data needed to understand the large space of $(m, n)$-orders in two and three dimensions, we ran these simulations on supercomputers at Northeastern University (Discovery cluster), Raman Research Institute, Université de Sherbrooke (MP2B cluster), and Simon Fraser University (Cedar cluster) over approximately 283.6 core-years. At a future date, the code used to perform these experiments will be merged with the Causal Set Generator [27] and the data will be published in the Encyclopedia of Quantum Geometries [28].

\section{Causal set dynamics in $\mathbf{d}=2,3$.}

We now present the results from simulations in $\mathbf{d}=2$ and $\mathbf{d}=3$ for the choice spacetimes, Eqn. 3. While we have performed simulations over several values of $n$ and non-locality parameter $\epsilon$ (see Eqn. 8 and 9), for the purpose of this work we fix $\epsilon=0.1$ and focus on the largest values of $n$ for which the simulations thermalise in reasonable time: $n=200$ for $\mathbf{d}=2$ and $n=300$ for $\mathbf{d}=3$. We choose $\alpha=4$, and $w=n^{1 /(\mathbf{d}-1)}$, so that $m=4 n^{2} \sim 1.6 \times 10^{5}$ in $\mathbf{d}=2$ and $m=4 n^{3 / 2} \sim 2.0 \times 10^{4}$ in $\mathbf{d}=3$.

To verify if our choice of $m$ is large enough, in each dimension we vary over different values of $m$ and run the MCMC simulation for two choices of $\beta \neq 0$. We find that the expectation values of the observables are roughly independent of $m$, as shown in Figs. 8, 9 , suggesting that the system is in the desired asymptotic regime.

\footnotetext{
${ }^{4}$ Here the index $i$ refers to the lattice point and not the direction.
} 
While the simulations can be carried out for larger values of $n$ while fixing $\beta$, the focus here is to study a range of $\beta$ to scan for phase transitions. We have generated data for several observables including the $\mathrm{BD}$ action, the ordering fraction, the height and the number of links. In the following analysis, we consider the raw data or the expectation value of these four observables. We have also computed the abundance $N_{i}$ of the $(i-2)$-element intervals in all cases, but have not exhibited their change as a function of $\beta$ explicitly, since there are too many of them to show. We will however use them to characterise the typical causal sets in the two phases in what follows. At every sweep, we also save the actual configuration on the lattice, so that we can record the changes in the configurations.

To begin, we check for ergodicity by choosing different initial configurations and find that the thermalised configurations are independent of this choice. In Fig. 10, we show a manifestation of this ergodicity in $\mathbf{d}=2$. The choices include the completely ordered $n$-element set, or chain, the set of unrelated $n$ elements, or antichain, the random $(m, n)$ order described in Sec.2, and the crown poset. As is evident, the thermalised value of the observables are independent of the initial configuration.

Next, we present the raw data for various observables for $\mathbf{d}=2,3$ for different values of $\beta$ in Fig. 11. These figures demonstrate very clearly that the configurations are thermalised at different values of $\beta$, except in a range of intermediate values. There is a clustering of the observables for small $\beta$ ("hot" configurations) and for large beta ("cold" configurations). In the intermediate range, however the observables fluctuate strongly. In $\mathbf{d}=2$, Fig. 11, we see that for $\beta=2.344$, the observables oscillate between two values, while in $\mathbf{d}=3$, this happens around $\beta=2.0$. These fluctuations are characteristic of a first order phase transition and resemble the behaviour of the 2 -orders 21,25 . In $\mathbf{d}=2$, just by eyeballing Fig. 11 , we note that the ordering fraction for the hot phase remains close to the value of 0.88 expected from a causal set that is approximated by the flat cylinder spacetime. Thus, even without further analysis, the data is clearly consistent with a manifold-like phase for small $\beta$. As we will see this is indeed the case.

Next, we process the raw data by first finding the autocorrelation times. In Fig. 12, we show a typical autocorrelation plot for the cold and hot values of $\beta$ in $\mathbf{d}=2,3$. After finding the autocorrelation times from the raw data, and discarding the first few autocorrelation times, we calculate the expectation value of the observables and the standard error. These are shown in Fig. 13, making explicit our surmise from the raw data, that the system undergoes a phase transition at some $\beta=\beta_{c}^{(\mathbf{d})}$. For our simulations, $\beta_{c}^{(2)} \approx 2.344$, and $\beta_{c}^{(3)} \approx 1.980$, consistent with our expectations from the raw data.

As $\beta$ becomes much larger, one finds the expected critical slowing down, which means very large $\beta$ values become more expensive to study. Moreover, around the transition values of $\beta$, since thermalisation is poor, it is harder to extract reliable expectation values even for $10^{4}$ sweeps. The raw data shows that the sytem oscillates between the hot and cold states and hence, rather than a Gaussian, one has a double Gaussian. As $\beta$ increases one expects the larger peak to shift from the hot phase to the cold phase, given a sufficient number of 
sweeps. However, the $10^{4}$ sweeps in our simulations are not always sufficient to see this. Fig. 14 shows an example of this in $\mathbf{d}=2$ for two values $\beta$ near $\beta_{c}^{(2)}$. From our data, at the higher value of $\beta=2.332$, the system spends all the $10^{4}$ sweeps in the hot phase, while at the lower value $\beta=2.328$ it oscillates between the hot and cold phases. This explains the lack of consistency in the data in Fig. 13 around the phase transition.

It is also possible to calculate the specific heat $\mathcal{C}$ as a function of $\beta$, where

$$
\mathcal{C}=\beta^{2}\langle S-\langle S\rangle\rangle^{2}
$$

$\mathcal{C}$ can be seen to develop a peak around the phase transition, but again, because of poor data in this region, we set aside this analysis for now.

In order to gauge the nature of a typical configuration, we show examples of causal sets that have been generated in the MCMC simulations in the hot and cold phases, in Figs. 15 and 16. In both $\mathbf{d}=2,3$, the causal set in the hot phase is clearly manifold-like in its random distribution, while in the cold phase it is layered and non-manifold-like. The number of layers in the cold phase in both $\mathbf{d}=2,3$ is approximately 5 , with most of the elements being distributed in the middle three layers. It is also clear from these figures that each element in a given layer is related to all the elements in the layer just above it and the one below it.

In Fig. 17, we show the expectation values of the various interval abundances in the hot and cold phases for $\mathbf{d}=2$ and $\mathbf{d}=3$. This distribution is drastically different in the two phases. In the hot phase, it is a reflection of the manifold-like properties of the causal set with, in particular, the interval abundances decreasing monotonically with $k$, the size of the intervals [29]. In the cold phase, on the other hand, the number of links is very large and the distribution develops "oscillations", with distinct peaks at very large values of $k$. The abundance of intervals of size $k=54$ in $\mathbf{d}=2$ and $k=89$ in $\mathbf{d}=3$, for example correspond approximately to the size of one of the layers. Such intervals lie between the layers $i-1$ and $i+1$. The next peak is sharper and higher and likely corresponds to the intervals between $i=2$ and $i=4$, suggesting that the central layer $i=3$ is the densest.

Fig. 18 illustrates the change in the occupancy of each constant time slice in the lattice, from a random manifold-like order to a layered order. For $\beta<\beta_{c}^{(\mathbf{d})}$ the occupancy is uniform, whereas for $\beta>\beta_{c}^{(\mathbf{d})}$ it is concentrated on very few layers. As noted above, the average number of layers in the second phase for $\mathbf{d}=2, \beta=3.2$ is $\sim 5$, and we observe similar behavior for $\mathbf{d}=3, \beta=3.0$. The occupancy is not uniform and tends to be largest in the middle layer, and smallest at the bottom-most and the top-most layer.

The functions $f_{2,3}(k, \varepsilon)$ in Fig. 20 which appear in the BD action, Eqn. (8) give a hint about the factors at play. We note that the action contribution will be larger if there are only $k$-intervals for which $f_{2,3}(k, \varepsilon)>0$. This is reflected by the abundance of intervals in Fig. 17, though the appearance of tertiary and higher peaks is a result of remnant entropic factors. 
The simulations slow down considerably for larger $\beta>\beta_{c}^{(\mathbf{d})}$, and hence the data becomes unreliable. However, one finds a clear trend as $\beta$ increases in Fig. 13, suggesting that the observables have not reached their asymptotic, zero temperature values.

In the zero-temperature phase we expect the action to completely dominate the entropy, which, as argued in [25], is the symmetric maximally connected bilayer poset. Such posets have approximately equal number of elements $\sim n / 2$ in the two layers, with every element in the first layer related to every element in the second layer, so that the number of links is $\sim n^{2} / 4$ (and the ordering fraction is $\sim 0.5$ for large $n$.). The symmetric maximally connected bilayer poset is the unique $n$-element poset in $\mathcal{E}_{\mathbf{d}}^{(m, n)}$ which maximizes the number of links. The BD action in both $\mathbf{d}=2$ and $\mathbf{d}=3$ thus takes its smallest value for these bilayer posets (Eqn. (8.9), which hence dominates the partition function. The maximally connected bilayer poset can be thought of as the "ground state" or zero-temperature state of this theory.

We find that our data is consistent with this expectation. In Fig. 19, we use fitting functions in the cold phase to model our data for each of the observables in $\mathbf{d}=2$. For $n=200$, the number of links in the symmetric bilayer poset is $10^{4}$, while the BD action is -360 . This is consistent with the fitting functions we find. For the $\mathbf{d}=3$ case with $n=300$, the expectation value of the number of links is $\sim 22,000$, and the BD action is $\sim-380$. From Fig. 13, we note that this is consistent with the data. 

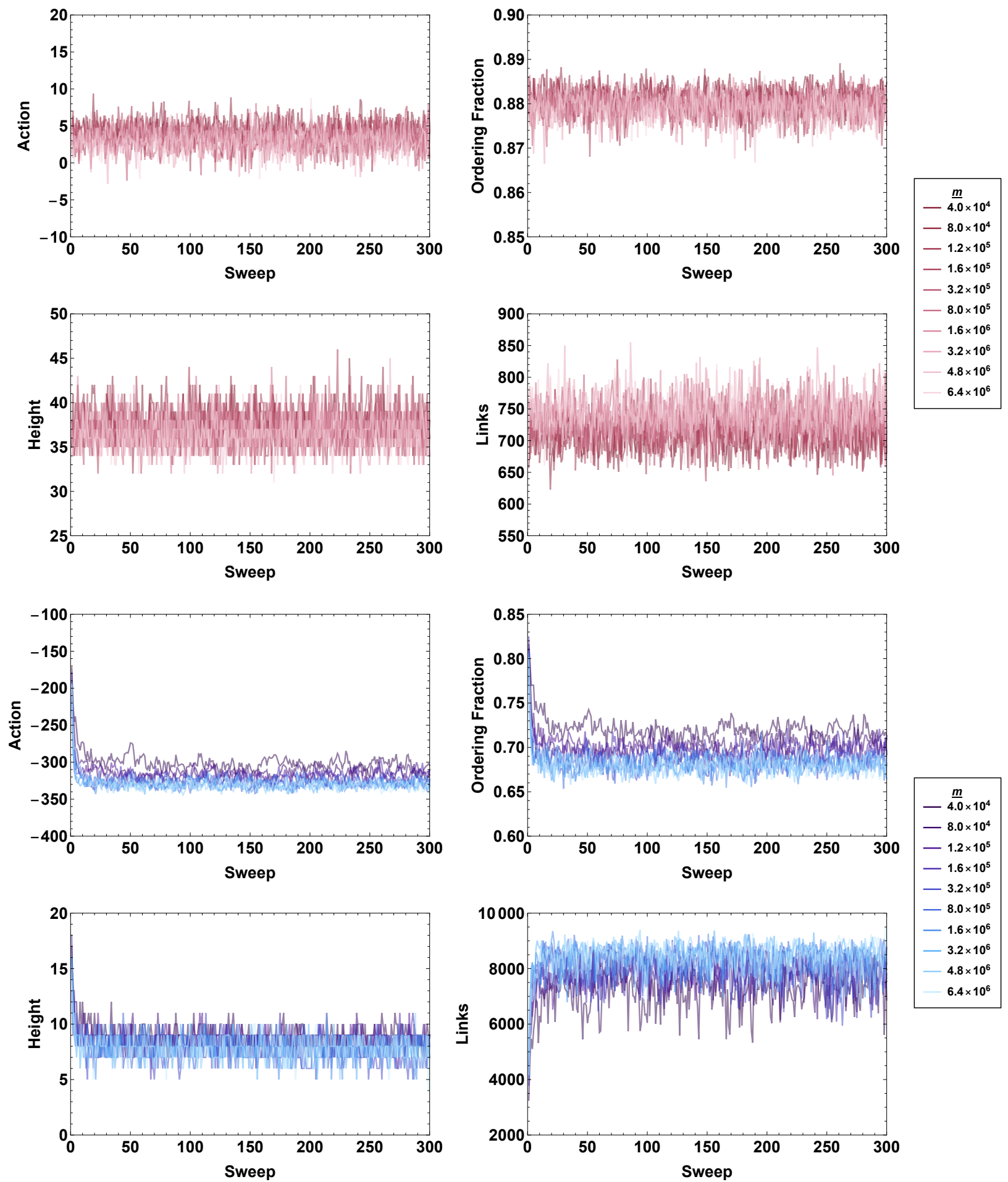

Figure 8: Raw data for the action, the ordering fraction, the height, and the number of links for $\mathbf{d}=2, n=200, \alpha=4, \epsilon=0.1$ and $\beta=0.8$ (upper four panels) and $\beta=3.2$ (lower four panels). 

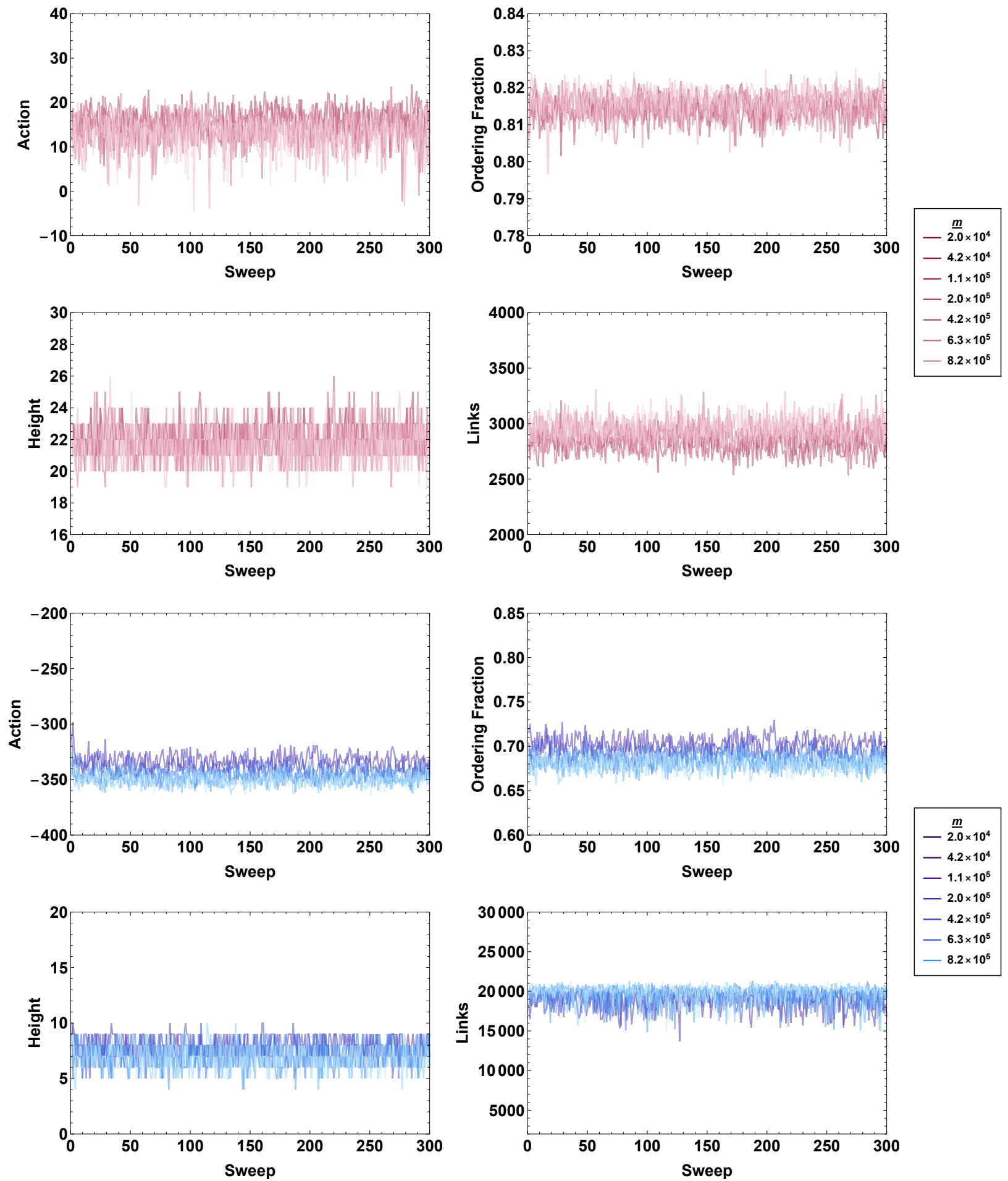

Figure 9: Raw data for the action, the ordering fraction, the height, and the number of links for $\mathbf{d}=3, n=300, \alpha=4, \epsilon=0.1$, and $\beta=1.0$ (upper four panels) and $\beta=3.0$ (lower four panels). 

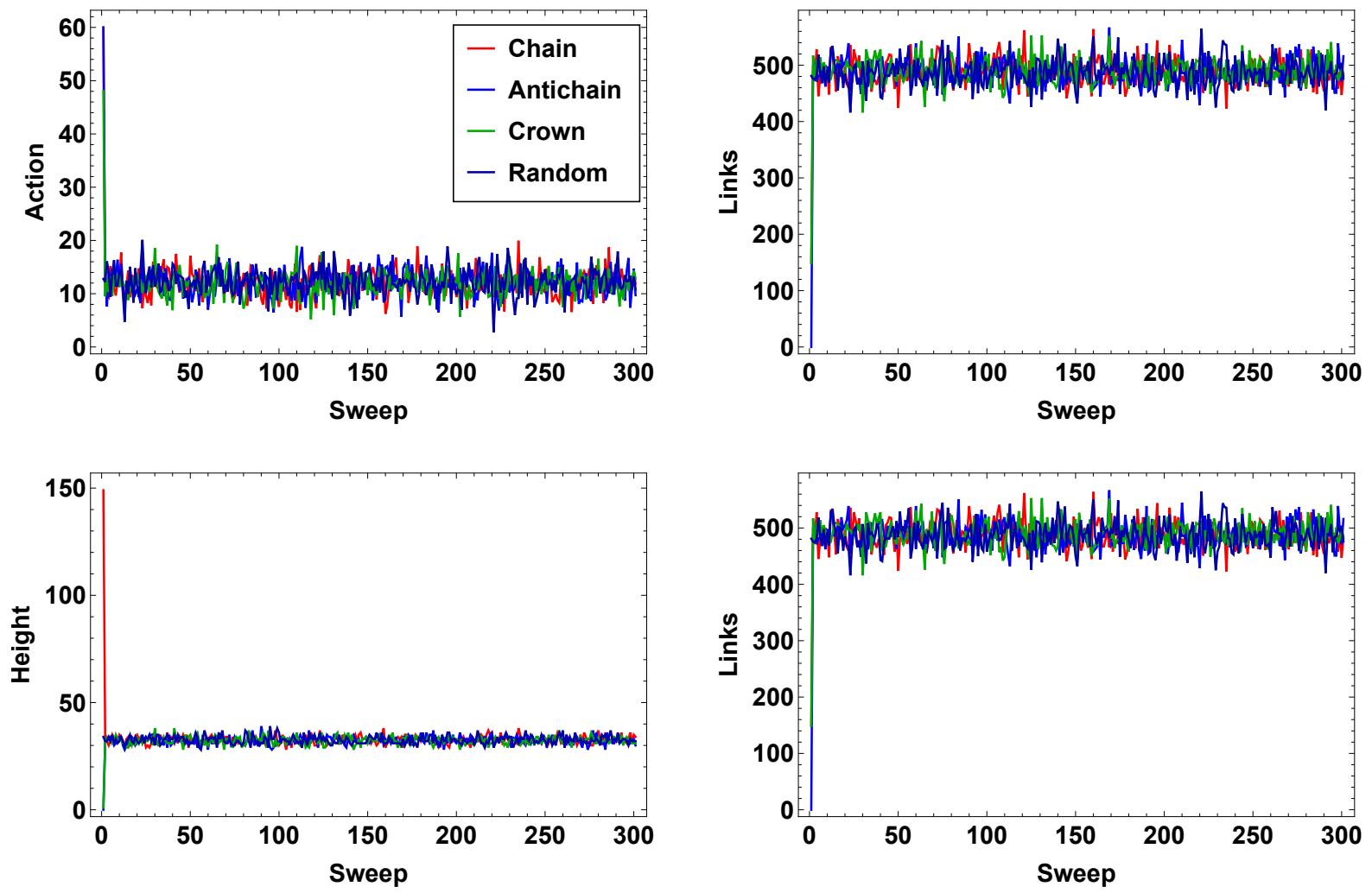

Figure 10: The observables per sweep for $\mathbf{d}=2, n=150, \epsilon=0.1, \beta=0.2$, and $\alpha=4$ for different initial conditions. 

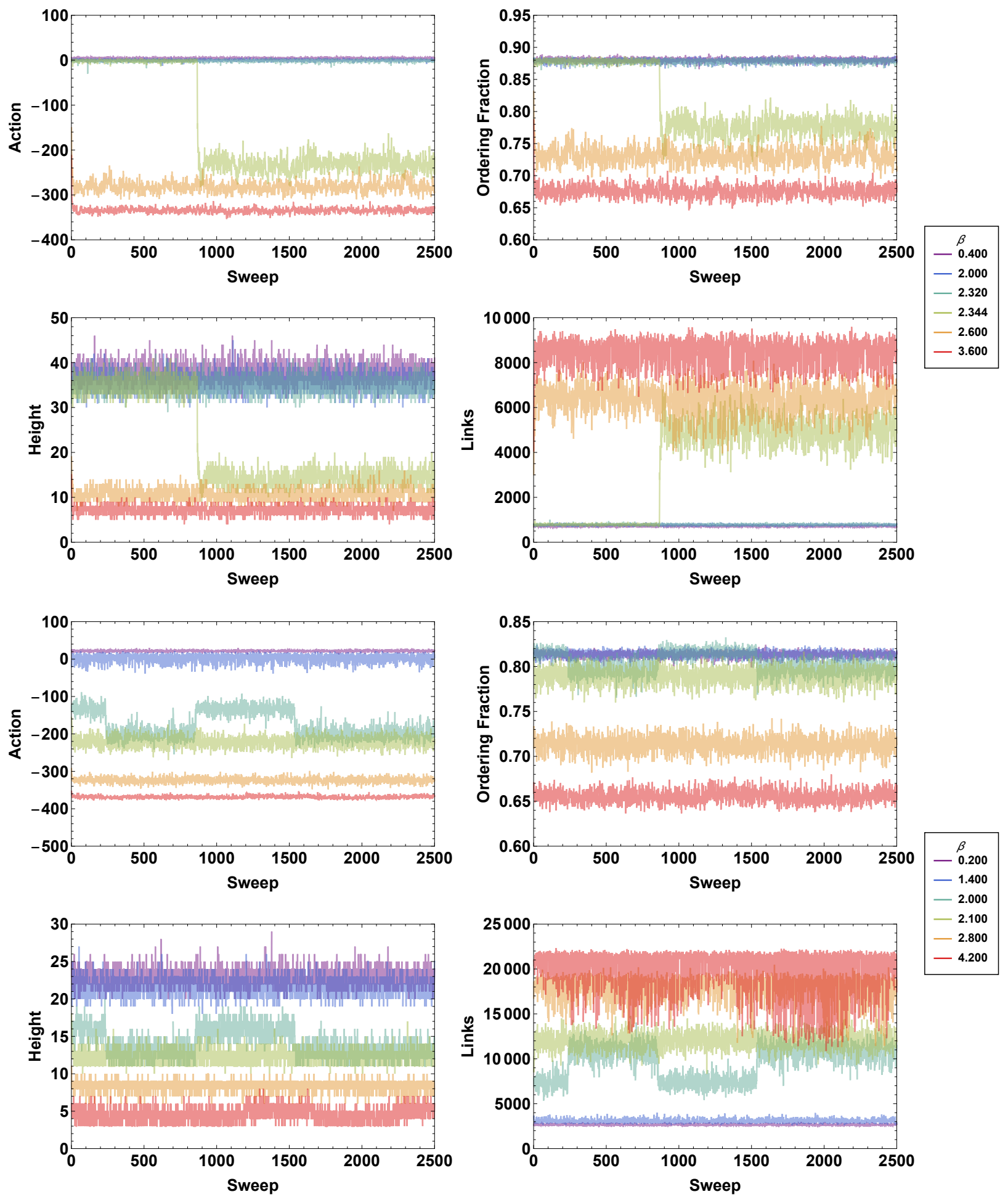

Figure 11: Raw data for the action, the ordering fraction, the height, and the number of links for $\epsilon=0.1$ and $\mathbf{d}=2, n=200$ (upper four panels) and $\mathbf{d}=3, n=300$ (lower four panels). 

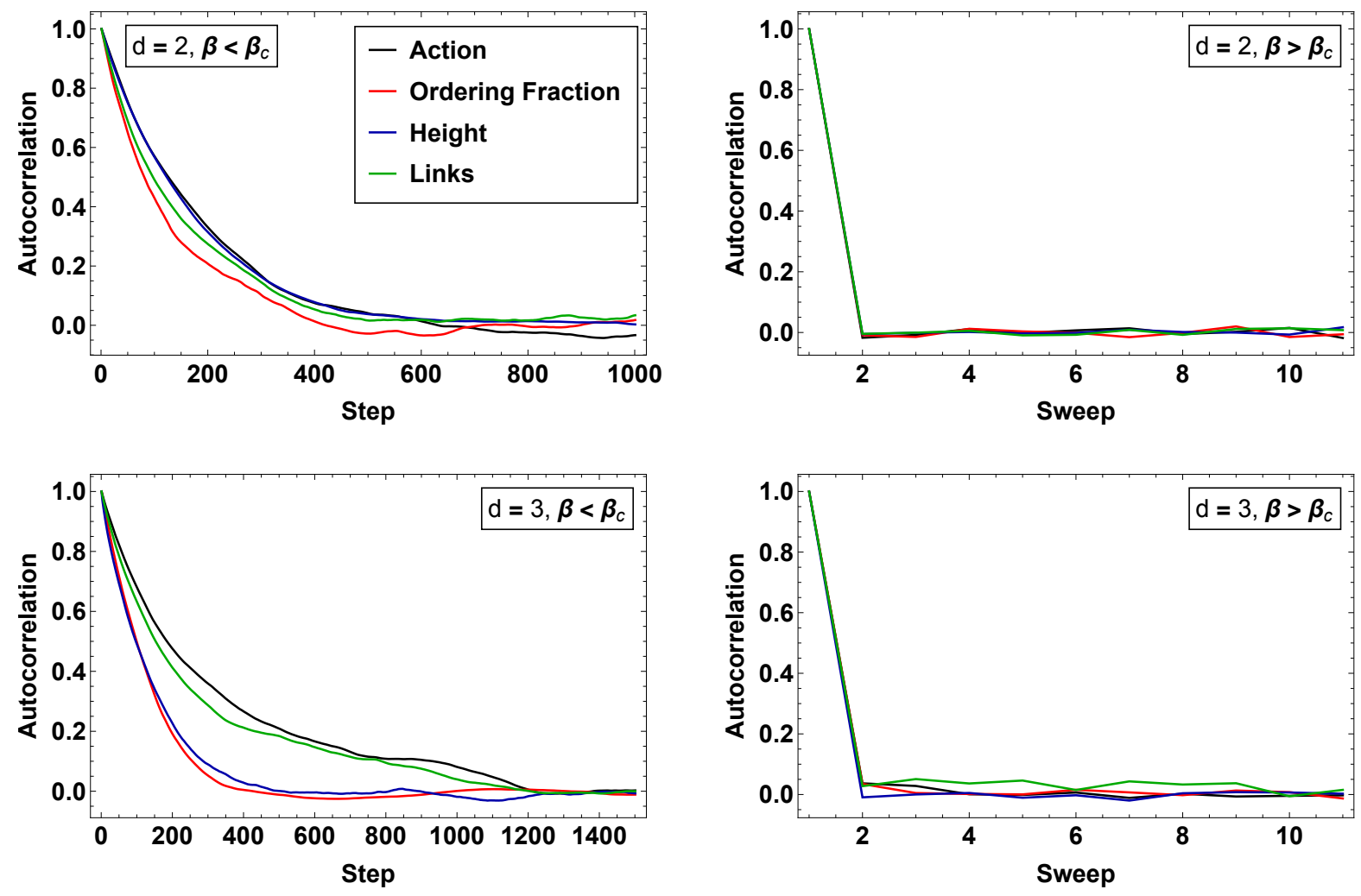

Figure 12: Examples of autocorrelation function for various observables for the hot $\left(\beta<\beta_{c}^{(\mathbf{d})}\right)$ and cold $\left(\beta>\beta_{c}^{(\mathbf{d})}\right)$ phases in $\mathbf{d}=2,3$. 

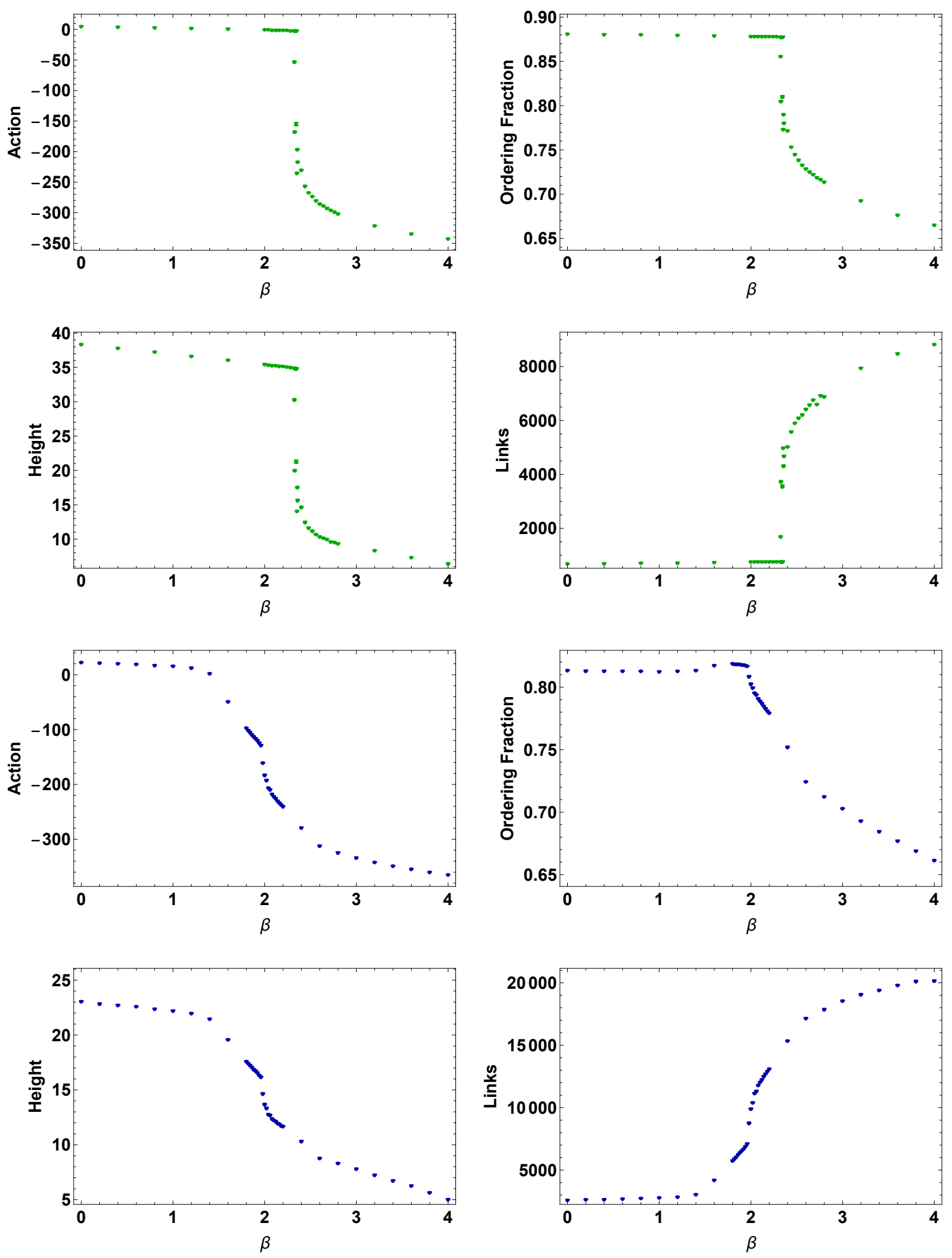

Figure 13: The expectation value of various observables as a function of $\beta$ for $\mathbf{d}=2$ (upper four panels) and $\mathbf{d}=3$ (lower four panels). The error bars are shown but are very small. 


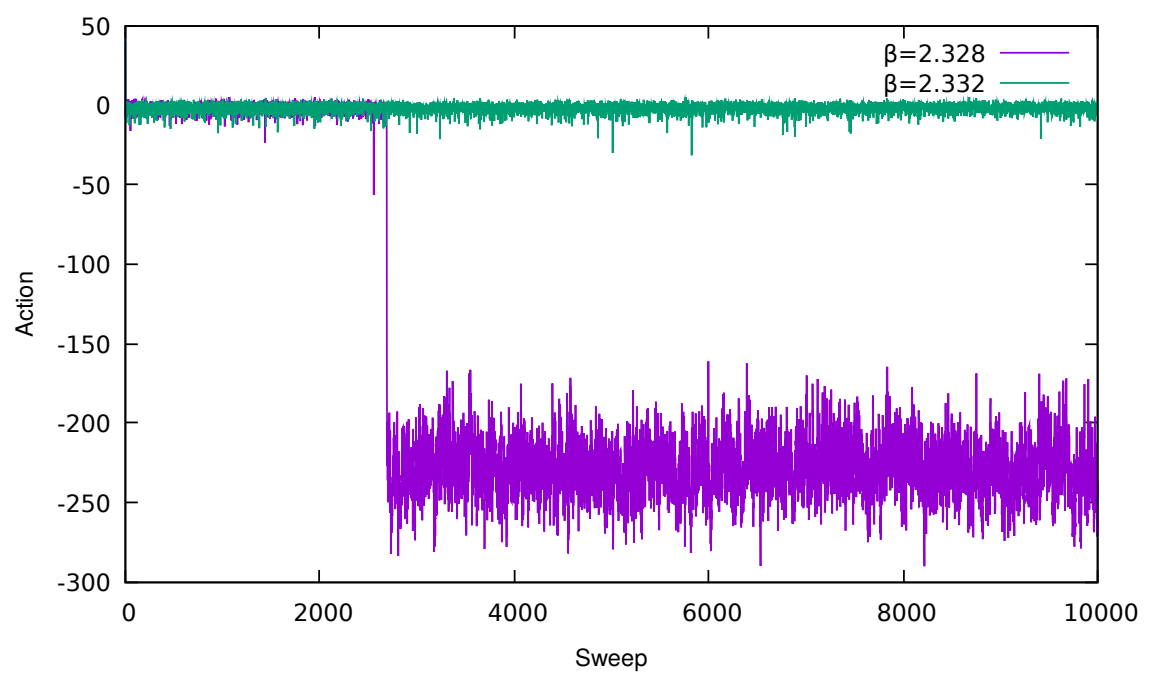

Figure 14: Raw data for the action in $\mathbf{d}=2$, showing the poor thermalisation near $\beta_{c}^{(2)}$ for $10^{4}$ sweeps. The system spends a longer time in the hotter phase at a lower temperature $\beta^{-1}=2.332^{-1}$ than at the higher temperature $\beta^{-1}=2.328^{-1}$. 

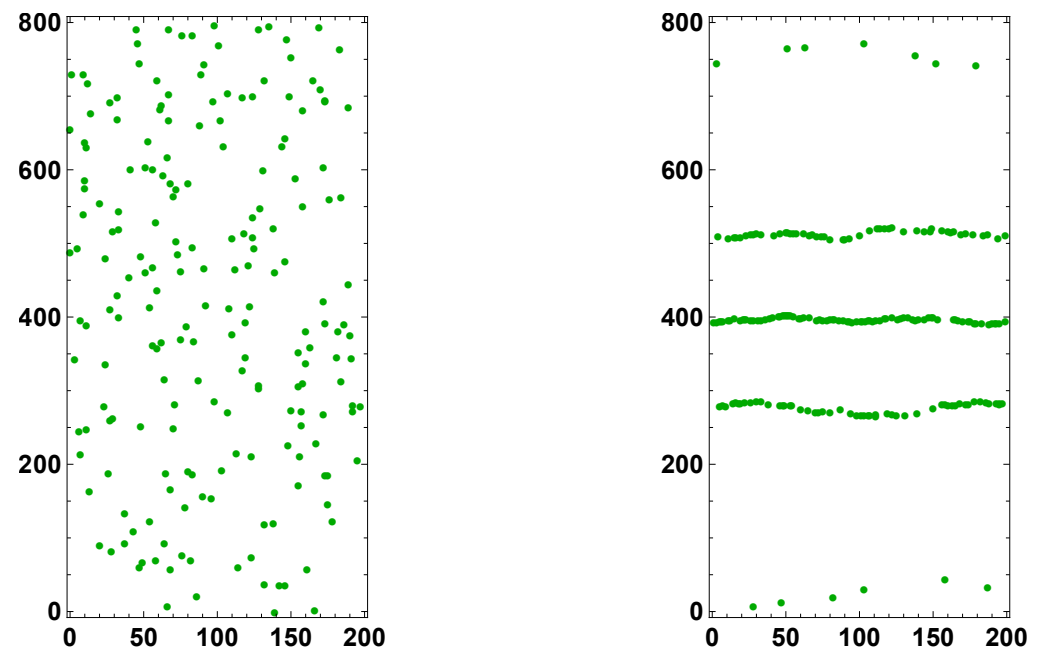

Figure 15: An example of a $\mathbf{d}=2$ configuration for $\beta=0.8$ (left) and $\beta=3.2$ (right).
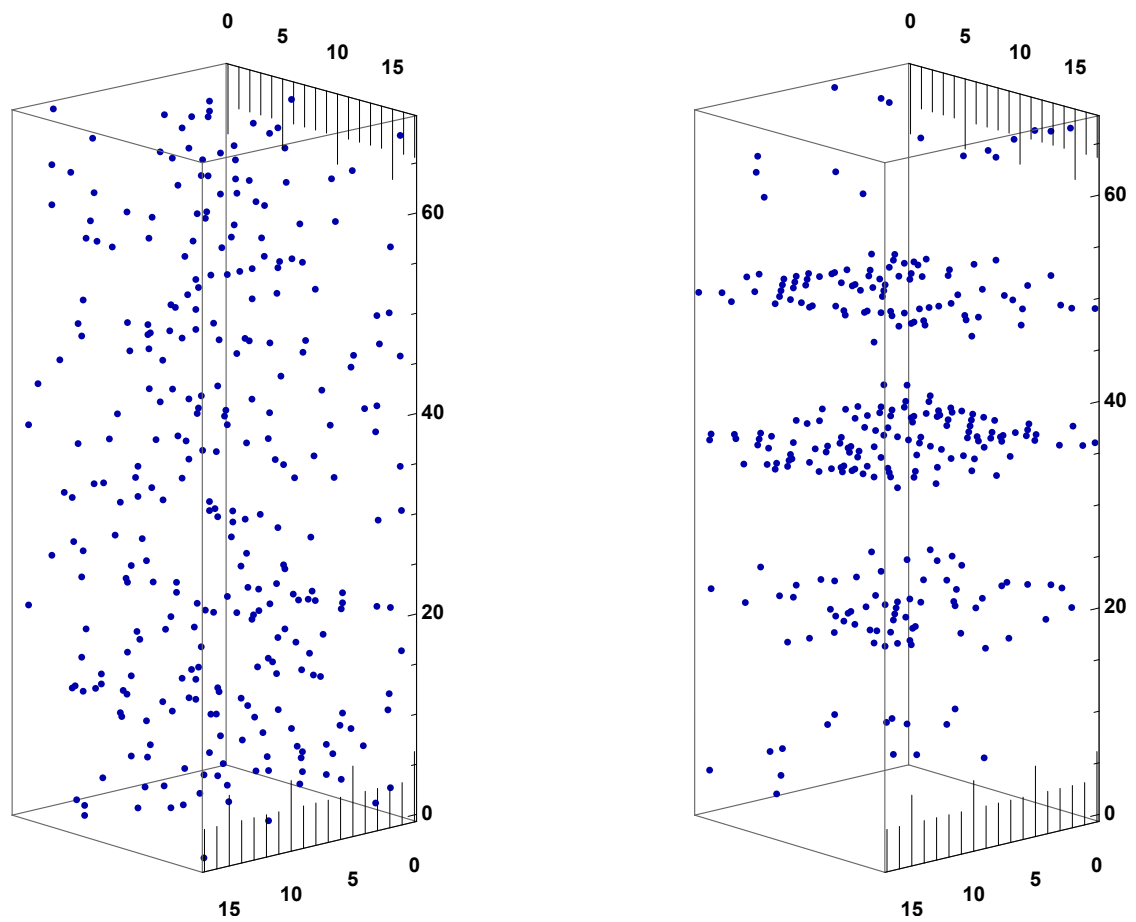

Figure 16: An example of a $\mathbf{d}=3$ configuration for $\beta=1.0$ (left) and $\beta=3.0$ (right). 

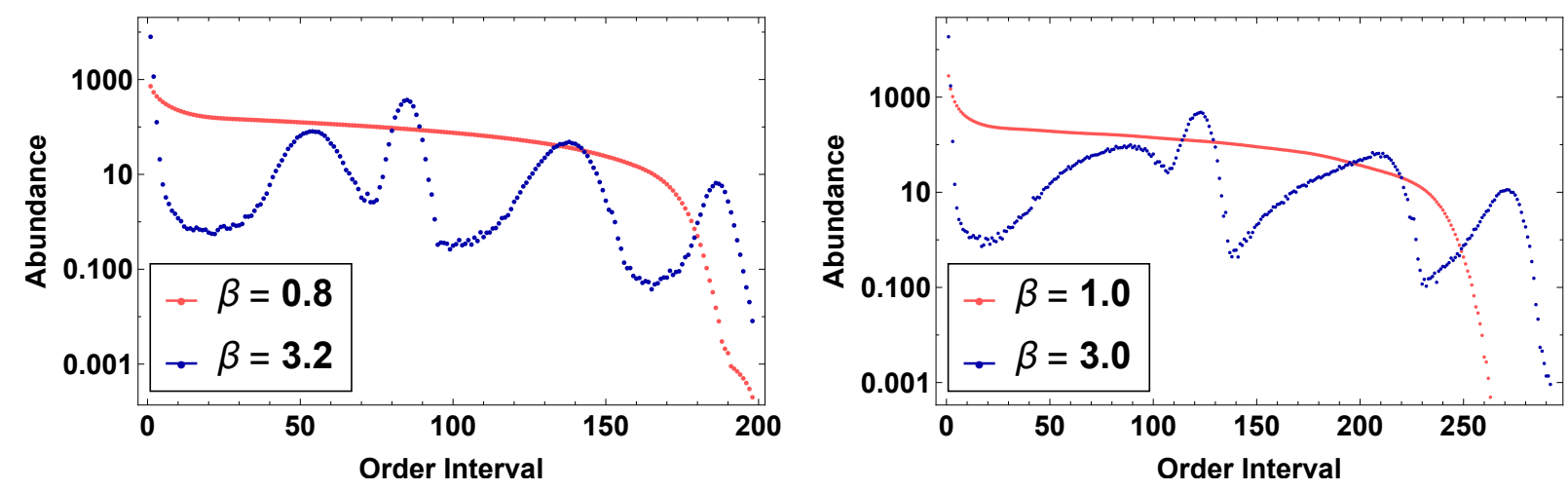

Figure 17: The interval abundances for the two phases in $\mathbf{d}=2$ (left) and $\mathbf{d}=3$ (right).
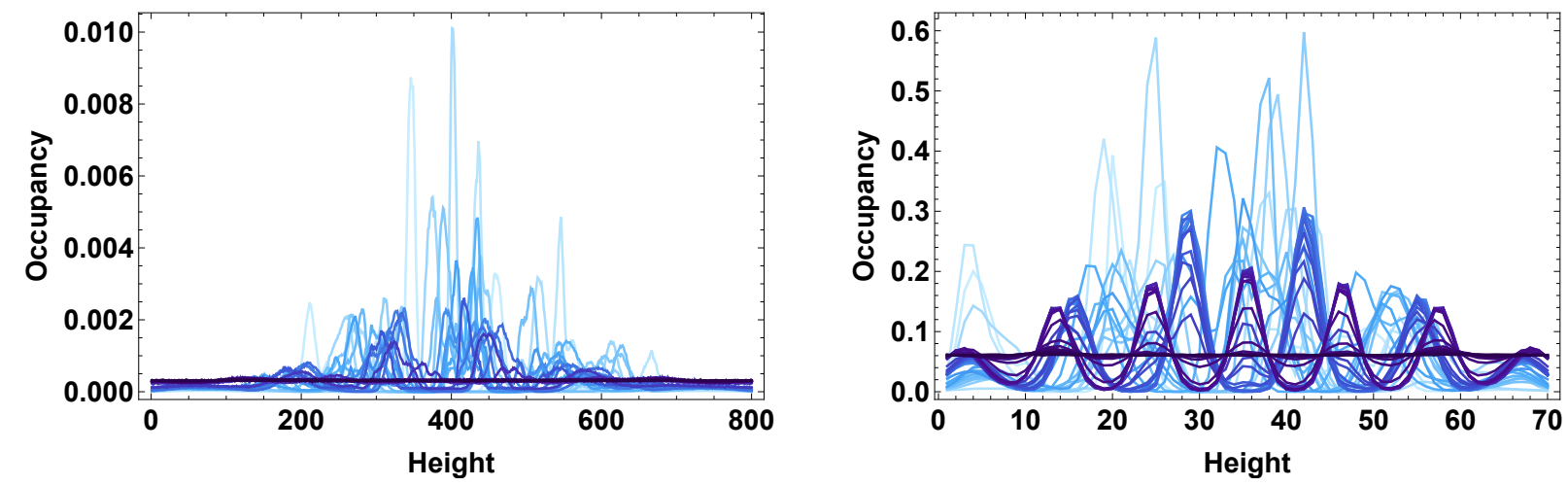

Figure 18: Occupancy or the number of elements at each time slice, for different values of $\beta$ for $\mathbf{d}=2, n=200$ (left) and $\mathbf{d}=3, n=300$ (right). In both cases we take $\epsilon=0.1$ and average over 50 causal sets each 200 sweeps apart. 

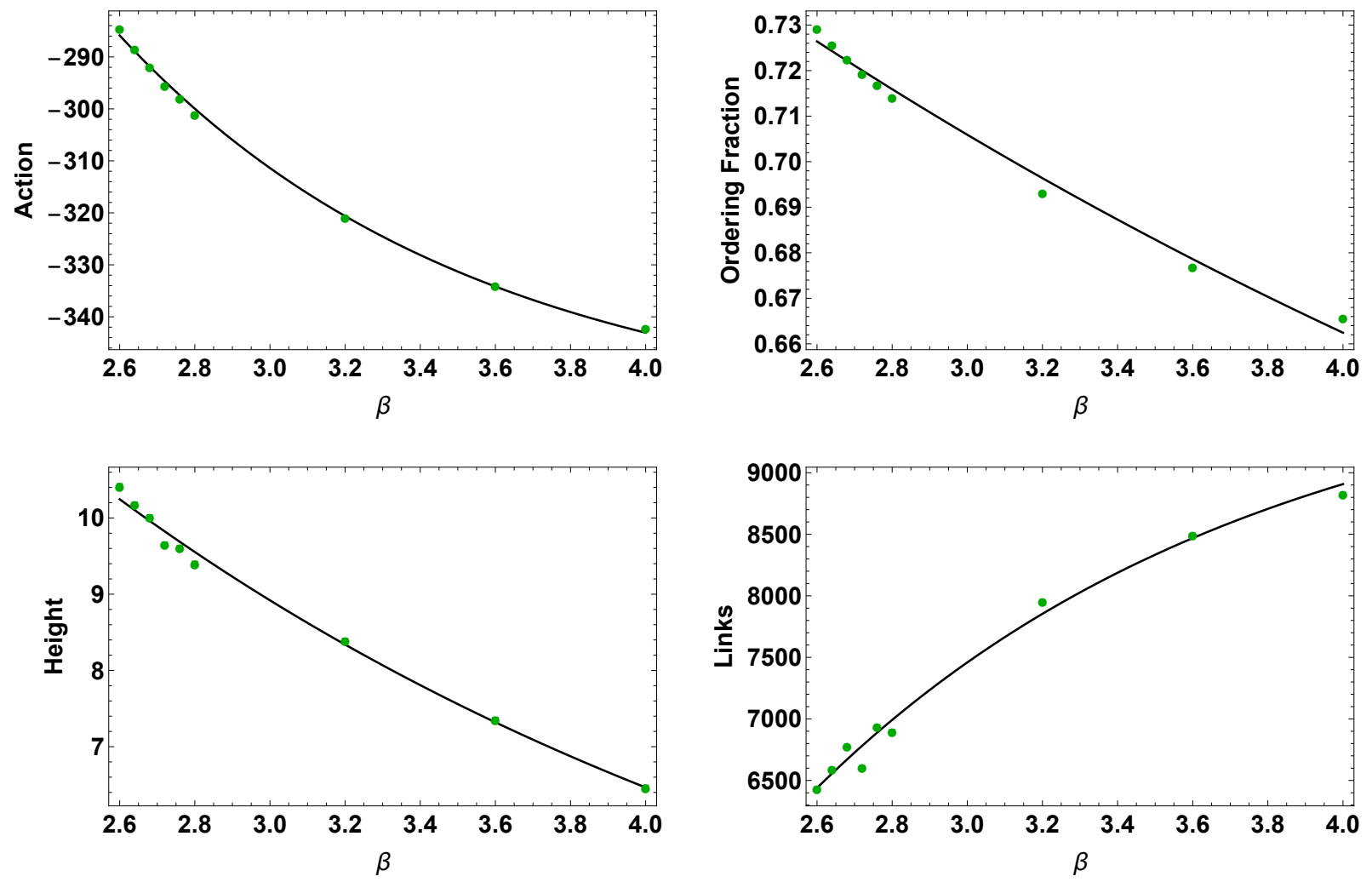

Figure 19: The fitting functions take the form $f(\beta)=A+B \exp (-C \beta)$, where the asymptotic value $A$ is set to be the value of the corresponding observable for the bilayer poset. 


\section{Conclusions}

The results presented in this work are a first step towards a full dimensional restriction of causal set theory, and are the first explicit explorations of non-trivial global spatial topology in $\mathbf{d}=2,3$.

For the theory defined by the partition function $\mathcal{Z}_{n, m}^{(\mathbf{d})}(\beta)$, Eqn. 6 , we see that as $\beta$ varies, there is a phase transition from a manifold-like phase to a non-manifold-like phase, which represents the battle between entropy and the action, and is similar to that observed for the 2 -orders [21, 25]. The BD action in both $\mathbf{d}=2,3$ is such that the measure is maximised by increasing the total number of links, which means that the ground state $($ or $\beta \rightarrow \infty)$ is the symmetric maximally connected bilayer poset. For smaller values of $\beta$ where the entropy-action battle is more subtle, however, there are interesting features which emerge.

The most obvious question that arises in this work is the role played by the choice of continuum spacetime $(M, g)$ in setting up the partition function $\mathcal{Z}_{n, m}^{(\mathbf{d})}(\beta)$. It is clear that the continuum phase is dominated by this geometry, whereas the layered phase seems largely independent of it. Importantly, the choice of $(M, g)$ introduces a preferred relative measure between manifold-like causal sets in $\Omega_{\mathbf{d}}^{(m, n)}$, since those that are approximated by $(M, g)$ are far more numerous than those that are not. Thus, at best, $\mathcal{Z}_{n, m}^{(\mathbf{d})}(\beta)$ represents fluctuations about the causal sets $c \sim(M, g)$, given that such a restriction is meaningful.

We have demonstrated that the choice of lattice size $m$ is not relevant as long as $m$ is large enough. However we have chosen a specific, regular lattice, and we don't yet know whether our results will change with a different lattice geometry. A more natural choice for the background lattice is a random $m$ element causal set obtained via a sprinkling into $(M, g)$. It would be important to explore simulations on such lattices and compare with our current results.

In order to obtain a more complete dimensional restriction, one would need to vary over all geometries with a given global topology and subsequently, vary over all topologies. By allowing the lattice $\mathcal{L}^{(m)}$ to vary, the relative weights should resemble that expected from the continuum. Work in this direction is currently underway [30]. Varying over topologies is trickier but preliminary investigations in this direction have also begun.

We have also restricted our analysis here to fixed values of $n$, without addressing the question of how robust the results are in the asymptotic or large $n$ limit. As shown in [25] using a finite sized scaling analysis for the $2 \mathrm{~d}$ orders, one must look for scaling behaviour with $\beta, n$ and $\epsilon$. Work is currently being done towards this goal [31].

Finally, as in all related work [21, 24, 25], there is the question of the analytic continuation and the physical significance of $\beta$ in full Lorentzian quantum gravity. This remains an important open question. Clearly the entropy-action struggle plays out differently for the quantum path integral as shown in [19]. There, a range of parameters was found for which the bilayer poset is suppressed. Relating such quantum results to the current statistical ones 
would be useful while exploring different analytic continuations. The hope is that further explorations of the sample space of causal sets and different analytic continuations will help us eventually arrive at a deeper understanding of these questions.

\section{Acknowledgements}

Research at Perimeter Institute is supported in part by the Government of Canada through the Department of Innovation, Science, and Economic Development Canada and by the Province of Ontario through the Ministry of Economic Development, Job Creation, and Trade. S. Surya is supported in part by a Visiting Fellowship at the Perimeter Institute. This research was enabled in part by support provided by the Discovery cluster at Northeastern University (www.northeastern.edu/rc), the MP2B cluster operated by Calcul Québec (www.calculquebec.ca), the Cedar cluster operated by WestGrid (www. westgrid.ca), Compute Canada (www.computecanada.ca), and Raman Research Institute (www.rri.res.in). We would like to thank Lisa Glaser for discussions during the later part of this work. 


\section{A Some Definitions for CST}

This section contains the definitions of various standard terms in CST that have appeared in the preceding sections.

- A causal set $c$ is said to causally embed into a spacetime $(M, g)$ if there exists an injection $\varphi: c \hookrightarrow(M, g)$ such that $e_{1} \prec e_{2} \leftrightarrow \varphi\left(e_{1}\right) \prec \varphi\left(e_{2}\right)$ for all $e_{1}, e_{2} \in c$. If the causal embedding is further obtained via a Poisson-sprinkling, then $c$ is said to be faithfully embedded into $(M, g)$, i.e., $c$ is said to be approximated by the continuum spacetime, (denoted $c \sim(M, g)$ ) and is hence manifold-like. Importantly, not all causal sets that causally embed into $(M, g)$ are manifold-like.

- A sample space is a collection of causal sets. In this work we refer to the following sample spaces: $\Omega_{n}$, the set of all $n$-element causal sets, $\Omega_{n}(M, g)$ the set of all $n$-element causal sets that causally embed into $(M, g)$ and $\Omega_{\mathbf{d}}^{(m, n)}$ the set of all $n$ element causal sets that embed into the lattice $\Omega_{\mathbf{d}}^{(m, n)}$.

- An order invariant for a causal set is a function on $c$ (over any field) which is independent of relabellings of $c$. In all the examples in this work, the functions considered are only over the field $\mathbb{R}$. The order invariance of a quantity (for example the measure) therefore means that it is independent of a relabelling of the causal sets.

- An $n$ element chain is a completely ordered $n$-element set $c$, i.e., for every $e_{i}, e_{j} \in c$, either $e_{i} \prec e_{j}$ or $e_{j} \prec e_{i}$. An $n$-element antichain is a set of mutually unrelated elements: $e_{i} \nprec e_{j} \forall e_{i}, e_{j} \in c$.

- A layer in a causal set $c$ is an antichain of elements with equal proper time, $\tau\left(e_{i}\right)$, defined as the longest chain from $e_{i}$ to any minimal element in the causal past of $e_{i} !^{5}$

- A crown poset $c$ is an $n=2 m, m \geq 3$ element poset with exactly two non-intersecting equal sized antichains $E_{1,2}$, such that $c=E_{1} \cup E_{2}$. Moreover, $c$ admits a labelling such that $\forall i(\bmod ) m, e_{i}^{1} \prec e_{i}^{2}, e_{i+1}^{2}$, are the only relations, with $e_{i}^{1} \in E_{1}, e_{i}^{2}, e_{i+1}^{2} \in E_{2}$. A crown poset causally embeds into any spacetime with a spatial non-contractible $S^{1}$ factor.

- The interval $I\left[e_{i}, e_{j}\right]$ in a causal set $c \ni e_{i}, e_{j}$ is the set of elements $e_{k} \in c$ such that $e_{i} \prec e_{k} \prec e_{j}$. Thus a $r$-element interval $I\left[e_{i}, e_{j}\right]$ contains $r$ "intervening" elements. For $e_{i} \prec e_{j}$, if $I\left[e_{i}, e_{j}\right]=0, e_{i}, e_{j} \in c$ are said to be linked. The abundance of $r$-element intervals $n_{r}$ in a causal set $c$ is simply the total number of $r$-element intervals.

- The ordering fraction in an $n$-element causal set is the ratio of the number of relations $r$ to the total number of possible relations on $n$-elements which is $\left(\begin{array}{l}n \\ 2\end{array}\right)$.

${ }^{5}$ In the low temperature phase of the partition function $\mathcal{Z}_{(n, m)}^{(\mathbf{d})}(\beta)$ where the action dominates over the entropy, we observe that causal sets self-assemble into layers in which nearly all elements are related to all those in adjacent layers (Figs. 15, 16. 


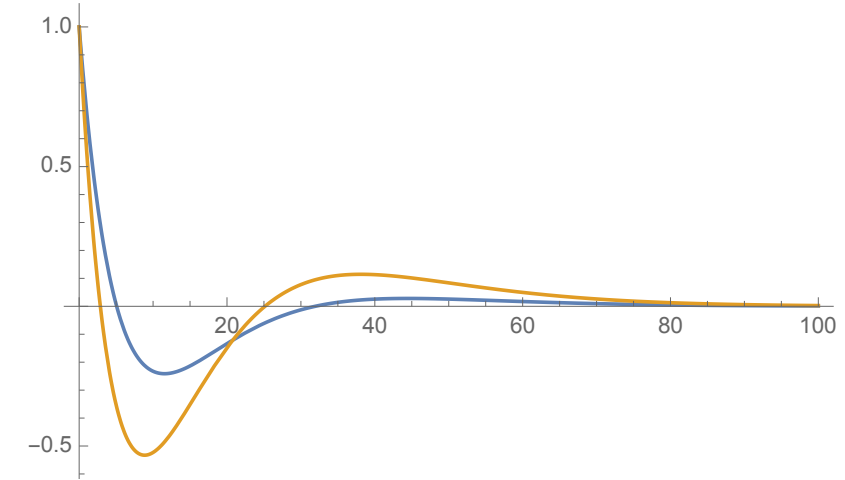

Figure 20: The functions $f_{2}(m, \epsilon)$ and $f_{3}(m, \epsilon)$ for $\epsilon=0.1$ as a function of the interval size. There are three consecutive bands of interval sizes, with alternating signs.

- The height of a causal set is the length of the longest chain in the causal set.

- The Benincasa-Dowker action is an order invariant which converges to the EinsteinHilbert action for causally convex compact regions of spacetime. In two and three dimensions, it is defined as

$$
\begin{aligned}
& S_{B D}^{(2)}(c) / \hbar=2 \epsilon\left[n-2 \epsilon \sum_{r=1}^{n-1} n_{r} f_{2}(r-1, \epsilon)\right], \\
& S_{B D}^{(3)}(c) / \hbar=\frac{1}{\Gamma(5 / 3)}\left(\frac{\pi \epsilon}{3 \sqrt{2}}\right)^{2 / 3}\left[n-\epsilon \sum_{r=1}^{n-1} n_{r} f_{3}(r-1, \epsilon)\right],
\end{aligned}
$$

where $f_{2,3}(r, \epsilon)$ are the smearing functions

$$
\begin{aligned}
& f_{2}(r, \epsilon)=(1-\epsilon)^{r}\left[1-\frac{2 r \epsilon}{1-\epsilon}+\frac{r(r-1) \epsilon^{2}}{2(1-\epsilon)^{2}}\right], \\
& f_{3}(r, \epsilon)=(1-\epsilon)^{r}\left[1-\frac{27 r \epsilon}{8(1-\epsilon)}+\frac{9 r(r-1) \epsilon^{2}}{8(1-\epsilon)^{2}}\right] .
\end{aligned}
$$

and $\epsilon=(0,1]$ is a non-locality scale, which is a free parameter in the theory.

- An $n$-element linear order $L=\left(l_{1}, \ldots l_{n}\right)$ over the set of $n$-integers $S=\{1, \ldots n\}$ is an automorphism of $S$ and is therefore totally ordered. An $n$-element poset $C$ is said to be a $d$-order if it is the "intersection" of $n$-element linear orders $\left\{L^{1}, \ldots, L^{d}\right\}$ : i.e., if its elements are given by $e_{j} \equiv\left(l_{j}^{1}, l_{j}^{2}, \ldots l_{j}^{d}\right)$, such that $e_{j} \prec e_{k}$ iff $l_{j}^{i}<l_{k}^{i}$ for all $i$. $C$ is then said to be of order-theoretic dimension $d$ and has a natural representation in the $d$-dimensional hypercube. The 2-orders (or 2d-orders) in particular, admit a simple representation with $L^{1,2}$ being the light-cone coordinates $\left\{\left(l_{i}^{1}=u_{i}, l_{i}^{2}=v_{i}\right)\right\}$ of the elements $e_{i}=\left(u_{i}, v_{i}\right)$ in a regular light-cone lattice in $\left(D_{2}, \eta\right)$. 


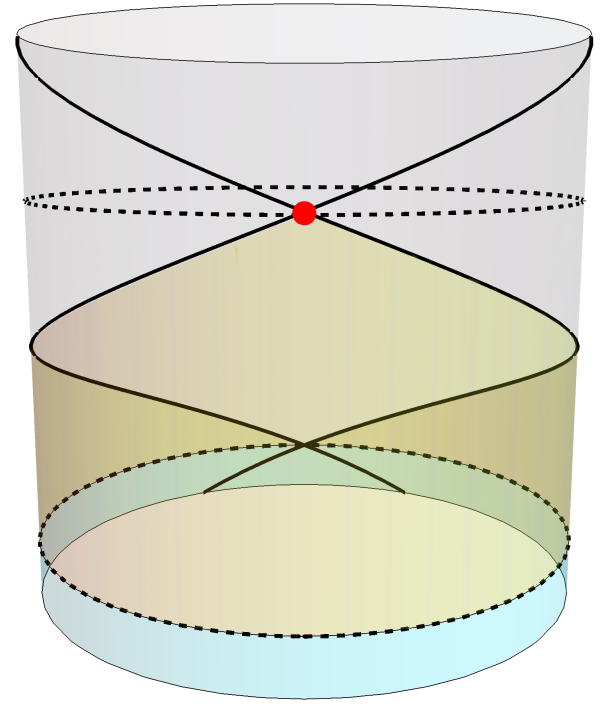

Figure 21: When the aspect ratio $\alpha>1 / 2$, the light-cone for an element can wrap all the way around the cylinder.

\section{B A calculation of the ordering fraction in $d=2$}

The ordering fraction in an $n$-element causal set is the ratio of the number of relations $R$ to the total number of possible relations $n(n-1) / 2$. For large $n, r \sim 2 R / n^{2}$. We now calculate the expectation value of $R$ for causal sets obtained via a Poisson sprinkling into the $\mathbf{d}=2$ cylinder spacetime $(M, g)$.

Consider the finite region $M$, where $M \sim S^{1} \times[0, h]$ for some choice of $h, w$, with $d s^{2}=-d t^{2}+w^{2} d \theta^{2}$, and the aspect ratio, $\alpha=h / w$. Let $\phi: c \hookrightarrow M$. Consider the event $p=(t, x)=\phi(e), e \in c$. The average number of events in $\phi(c)$ that are related to $p$ is given by the volume of the region $I^{+}(p) \cap M$ and $I^{-}(p) \cap M$. The average number of relations in the ensemble of causal sets that are approximated by $M$ is thus given by

$$
\langle R\rangle=\int_{M} d p \int_{q \prec p} d q=\int_{M} d p \operatorname{vol}\left(I^{-}(p) \cap M\right)
$$

where we need to consider only the past of $p, I^{-}(p) \cap M$, and we take $\rho=1$. While the ordering fraction for $\mathbb{M}^{d}$ is related to the continuum dimension via the Myrheim-Meyer dimension estimator, and is hence independent of the size of the region, this is not the case here. For $\alpha<1 / 2$, the light rays from $p$ do not wrap around the cylinder, and the result is similar to $\mathbb{M}^{2}$. For $\alpha>1 / 2$ this is not the case and we must split the past volume from $p$ into two different parts as shown in Fig. 21.

For $p=(t, x)$, with $t \leq w / 2, V(p) \equiv \operatorname{vol}\left(I^{-}(p) \cap M\right)=t^{2}$. The average number of such 
relations is therefore given by

$$
\int_{t=0}^{w / 2} d t \int_{0}^{w} d x t^{2}=w^{4} / 24
$$

For $p=(t, x), t \geq w / 2$, the average number of relations splits up into two parts, one with (a) $q=\left(t^{\prime}, x^{\prime}\right)$ such that $t^{\prime} \leq t-w / 2$ and one with (b) $t^{\prime} \geq t-w / 2$ (see Fig. 21). Thus the average number of relations for such $(p, q)$ is

$$
\int_{w / 2}^{h} d t \int_{0}^{w} d x\left(V_{a}(t)+V_{b}(t)\right)=\int_{w / 2}^{h} d t \int_{0}^{w} d x\left(w(t-w / 2)+w^{2} / 4\right)=w^{2} h^{2} / 2-w^{3} h / 4 .
$$

Adding these two expressions gives us the total expectation value for the number of relations

$$
\langle R\rangle=\frac{(h w)^{2}}{2}\left(1-\frac{1}{2 \alpha}+\frac{1}{12 \alpha^{2}}\right)
$$

or

$$
\langle r\rangle=\left(1-\frac{1}{2 \alpha}+\frac{1}{12 \alpha^{2}}\right)
$$

In our simulations in $\mathbf{d}=2$, we have used $\alpha=4$. This corresponds to an $\langle r\rangle \sim 0.88$, which is consistent with our observations.

\section{References}

[1] L. Bombelli, J. Lee, D. Meyer, and R. Sorkin, Phys. Rev. Lett. 59, 521 (1987).

[2] R. D. Sorkin, in Proceedings of the Valdivia Summer School, Series of the Centro de Estudios Cientificos de Santiago), edited by A. Gomberoff and D. Marolf (New York, Springer, 2005) gr-qc/0309009.

[3] F. Dowker, in 100 Years Of Relativity: space-time structure: Einstein and beyond, edited by A. Ashtekar (World Scientific, 2005) pp. 445-464, arXiv:gr-qc/0508109 [gr-qc]

[4] J. Henson, in Approaches to quantum gravity, edited by D. Oriti (Cambridge University Press, Cambridge, 2006) pp. 393-413, arXiv:gr-qc/0601121 [gr-qc].

[5] J. Henson, in Proceedings, Foundations of Space and Time: Reflections on Quantum Gravity: Cape Town, South Africa (2010) arXiv:1003.5890 [gr-qc].

[6] P. Wallden, Proceedings, 15th Conference on Recent Developments in Gravity (NEB 15): Chania, Crete, Greece, June 20-23, 2012, J. Phys. Conf. Ser. 453, 012023 (2013).

[7] S. Surya, Living Rev. Rel. 22, 5 (2019), arXiv:1903.11544 [gr-qc]. 
[8] S. Hawking, A. King, and P. McCarthy, J. Math. Phys. (1976).

[9] D. B. Malament, J. Math. Phys. 18, 1399 (1977).

[10] W. G. Unruh and R. M. Wald, Phys. Rev. D 40, 2598 (1989).

[11] R. D. Sorkin, Int. J. Theor. Phys. 36, 2759 (1997), arXiv:gr-qc/9706002 [gr-qc]].

[12] D. M. Benincasa and F. Dowker, Phys.Rev.Lett. 104, 181301 (2010).

[13] F. Dowker and L. Glaser, Class. Quantum Grav. 30, 195016 (2013).

[14] L. Glaser, Class. Quantum Grav. 31, 095007 (2014).

[15] D. J. Kleitman and B. L. Rothschild, Trans. Am. Math. Soc. 205, 205 (1975).

[16] D. Dhar, J. Math. Phys. 19 (1978).

[17] D. Dhar, Pacific J. Math. 90 (1980).

[18] A. Eichhorn, Class. Quantum Grav. 35, 044001 (2018), arXiv:1709.10419 [gr-qc].

[19] S. P. Loomis and S. Carlip, Class. Quantum Grav. 35, 024002 (2018), arXiv:1709.00064 [gr-qc] .

[20] G. Brightwell, J. Henson, and S. Surya, Class. Quantum Grav. 25, 105025 (2008).

[21] S. Surya, Class. Quantum Grav. 29, 132001 (2012).

[22] P. Winkler, Order 7, 329 (1991).

[23] M. H. El-Zahar and N. W. Sauer, Order 5, 239 (1988).

[24] J. B. Hartle and S. W. Hawking, Phys. Rev. D 28, 2960 (1983).

[25] L. Glaser, D. O'Connor, and S. Surya, Class. Quantum Grav. 35, 045006 (2018), arXiv:1706.06432 [gr-qc]].

[26] W. Cunningham and D. Krioukov, Comput. Phys. Commun. 233, 123 (2018).

[27] W. Cunningham, "Causal Set Generator,"] (2017).

[28] Zenodo Digital Library, "Encyclopedia of quantum geometries,"] (2019).

[29] L. Glaser and S. Surya, Phys. Rev. D 88, 124026 (2013), arXiv:1309.3403 [gr-qc]].

[30] W. Cunningham and S. Surya, "Flucutating lattices in CST," In progress.

[31] W. Cunningham, L. Glaser, and S. Surya, "Finite sized scaling analysis in $d=2,3$ CST," In progress. 